\title{
SDT 11. THE ORNL BENCHMARK EXPERIMENT FOR NEUTRON TRANSPORT THROUGH IRON AND STAINLESS STEEL, PART I
}

R. E. Maerker 


\section{DISCLAIMER}

This report was prepared as an account of work sponsored by an agency of the United States Government. Neither the United States Government nor any agency Thereof, nor any of their employees, makes any warranty, express or implied, or assumes any legal liability or responsibility for the accuracy, completeness, or usefulness of any information, apparatus, product, or process disclosed, or represents that its use would not infringe privately owned rights. Reference herein to any specific commercial product, process, or service by trade name, trademark, manufacturer, or otherwise does not necessarily constitute or imply its endorsement, recommendation, or favoring by the United States Government or any agency thereof. The views and opinions of authors expressed herein do not necessarily state or reflect those of the United States Government or any agency thereof. 


\section{DISCLAIMER}

Portions of this document may be illegible in electronic image products. Images are produced from the best available original document. 
This report was prepared as an account of work sponsored by the United States Government. Neither the United States nor the United States Atomic Energy Commission, nor any of their employees, nor any of their contractors, subcontractors, or their employees, makes any warranty, express or implied, or assumes any legal liability or responsibility for the accuracy, completeness or usefulness of any information, apparatus, product or process disclosed, or represents that its use would not infringe privately owned rights. 


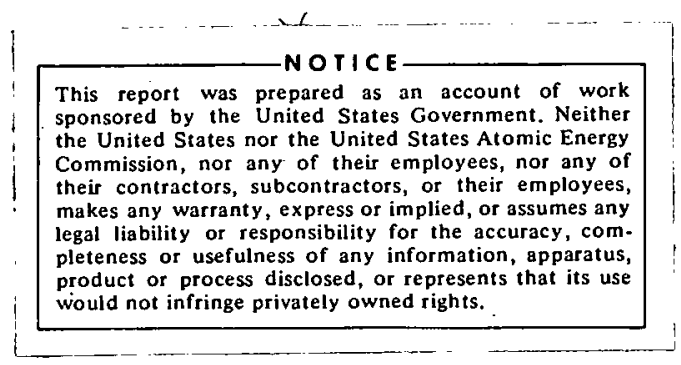

ORNL-TM-4222

(ENDF 188)

Contract No. W-7405-Eng-26

Neutron Physics Division

SIT11. THE ORNL BENCHMARK EXPERIMENT FOR NEUTRON TRANSPORT THROUGH IRON AND STAINLESS STEEL, PART I

R. E. Maerker

Reference: R. E. Maerker and F. J. Muckenthaler, "Final Report on a Benchmark Experiment for Neutron Transport Through Iron and Stainless Steel," ORNL-4892 (1974)

\section{SEPTEMBER 1974}

OAK RIDGE NATIONAL LABORATORY

Oak Rilge, Tennessée 37830

operated by

UNION CARBIDE CORPORATION

for the

U. S. ATOMIC ENERGY COMMISSION

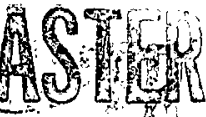


THIS PAGE

\section{WAS INTENTIONALLY}

LEFT BLANK 
TABLE OF CONTENTS

Page No.

ABSTRACT -

DESCRIPTION OF THE EXPERIMENT AND SOURCE DATA ---------------- 1

DATA OBTAINED BEHIND THE SLABS -

METHODS OF CALCULATION 


\section{THIS PAGE}

\section{WAS INTENTIONALLY \\ LEFT BLANK}


The first part of an experiment concerning deep neutron penetration in iron and stainless steel is described, and experimental results in a format for CSEWG shielding integral data testing are presented. These results provide a basis for verification of the accuracy of iron and stainless steel cross sections used in transport calculations. The experiment was performed at the Tower Shielding Facility of ORNL and included measurements of both the neutron fluence and neutron spectra behind slabs of iron up to $3 \mathrm{ft}$ thick and of stainless steel up to $1 \mathrm{ft}$ thick. 
DESCRIPTION OF THE EXPERIMENT AND SOURCE DATA

Both the top shield and the structural components in the design of a fast reactor, and the thermal shield of a conventional thermal reactor contain a large amount of iron in the form of carbon and stainless steels, and iron therefore constitutes an important part of the neutron shield. The carbon steels consist of relatively pure (i.e., 98-99\%) iron, while the stainless steels contain, in addition to about $70 \%$ iron, considerable amounts of chromium and nickel. Since these steel components have thicknesses of the order of $3 \mathrm{ft}$, it is essential that accurate experimental results be available to verify transport calculations for deep penetration of neutrons through iron and'stainless steel.

Consequently, a series of transmission measurements of neutrons above thermal energies through various thicknesses of iron slabs and also through a 12-in. stainless steel slab have been performed at the Tower Shielding Facility using a collimated beam of reactor neutrons as a source. These measurements were madè behind various combinations of thin (i.e., $\frac{1}{2}$ to $2 \mathrm{in}$. thick) $5 \mathrm{ft}$ by $5 \mathrm{ft}$ slabs; measurements were obtained behind iron thicknesses of approximately 1.5, 4,6,12, 24, and 36 in. Figure 1 shows a schematic of the experimental geometry for the 36-in. case.

The thickness, density, and composition of the individual slabs used in the experiment were accurately determined. The density of the type 304 stainless steel slabs averaged $7.86 \mathrm{~g} / \mathrm{cm}^{3}$ and the density of the iron slabs averaged $7.79 \mathrm{~g} / \mathrm{cm}^{3}$. The composition of the slabs is shown in Table I, where it is to be observed that the "iron" slabs were actually carbon (i.e., mild) steel.

Sufficient measurements of the incident neutron beam were made that an absolute energy spectrum from thermal to $10 \mathrm{MeV}$ could be obtained for use in calculations. This incident spectrum is presented in Table II in a 220-group structure. The intensities in Table II are for any point on the exit plane of the collimator located within a diameter of $6 \frac{1}{2}$ in. Mapping of the incident beam along the axial direction established the 


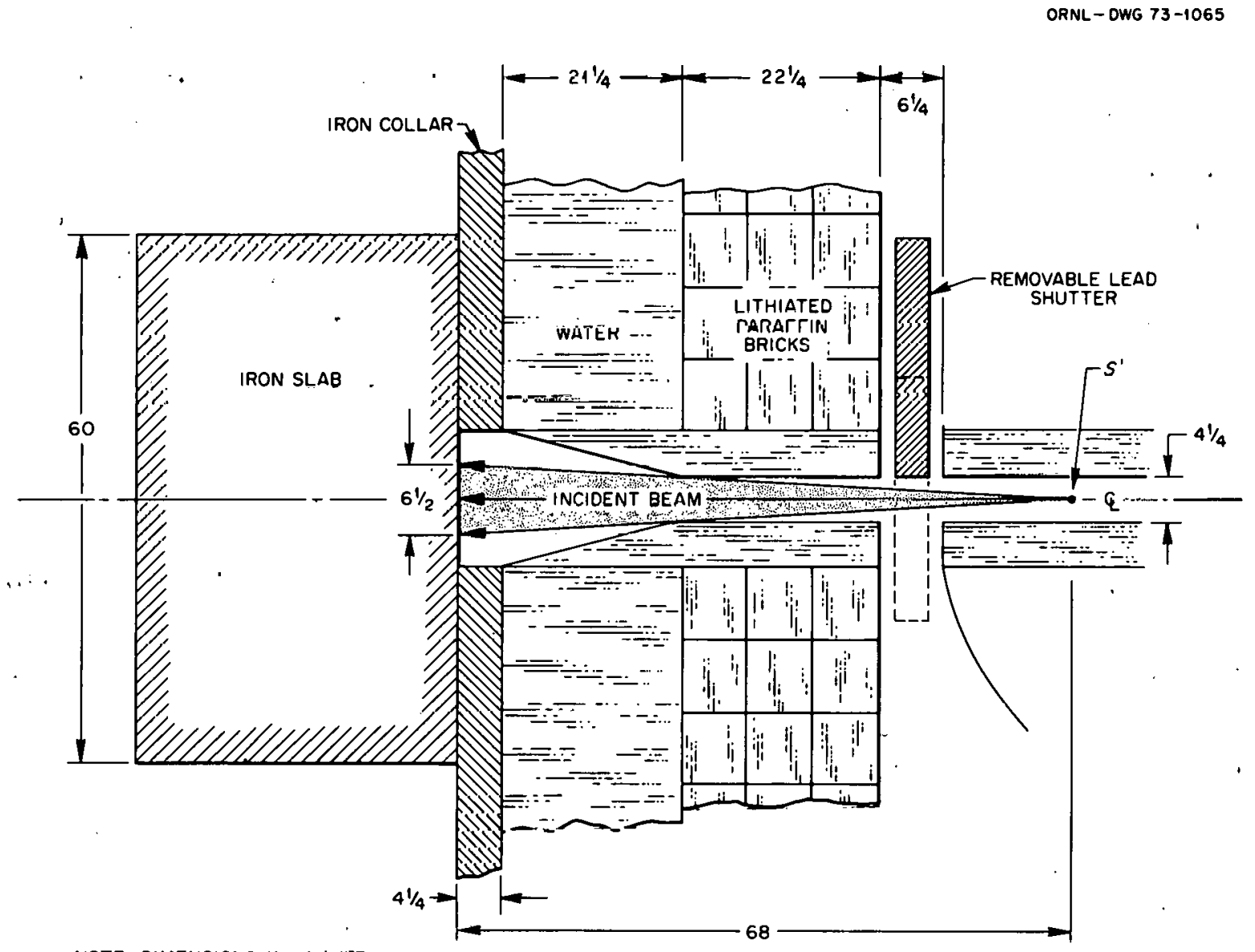

NOTE: DIMENSIONS IN INCHES

Hig. 1. Experimental Configuration for the 4-1/4-in.-diam Collimator with the 3-ft-Thick Iron Slab in Place. (This collimator was used for all the measurements except those made behind 18 in. of stainless steel.) 
fact that the tightly collimated source could be represented as a virtual point anisotropic source located 68 in. inside the collimator from the edge of the iron collar. (point $S^{\prime}$ in Fig. 1) with the beam intensity. uniform over a diameter of $6 \frac{1}{2}$ in. at the mouth of the collimator and zero elsewhere. The accuracy of the incident absolute spectrum in Table I-I is estimated to be $\pm 10 \%$ down to $200 \mathrm{keV}$ and $\pm 20 \%$ below $200 \mathrm{keV}$. The ratio of surface-integrated current over the collimator to centerline current is $212.5 \mathrm{~cm}^{2}$.

Neutron spectrum measurements beyond the iron or stainless steel slabs were taken using two types of spectrometers. These were: (I) an NE-213 liquid scintillator, which determines spectra in the energy range 0.8 to $10 \mathrm{MeV}$ with the aid of the unfolding code FERDOR; and (II) a Benjamin proton recoil spectrameter which determines spectra in the energy range 100 $\mathrm{keV}$ to $1.5 \mathrm{MeV}$ with the aid of the unfolding code SPEC4. Table III gives the resolution of the $\mathrm{NE}-213$ as a function of energy. The resolution of the Benjamin spectrometer is constant at 10\% FWHM. In addition, a set of spherical $\mathrm{BF}_{3}$ detectors surrounded by various thicknesses of polyethylene ( 0 to $5 \mathrm{in.}$ ) and an outside shell of cadmium were used to obtain weighted integral flux measurements. These Bonner ball detectors have response functions which peak in different regions of the spectrum. The composition of each Bonner ball and the location of the center of detection is listed in Table IV. The response function for each of the three Bonner balls in a 100 group GAM-II structure is presented in Tables $V$ through VII. They are expressed in units of counts $/ \mathrm{sec}$ per neutron $/ \mathrm{cm}^{2} / \mathrm{sec}$ uniformly incident over the outside hemispherical surface of the ball, and were obtained by adjoint ANISN calculations normalized to calibration experiments performed at the Tower Shielding Facility. The estimated accuracy of the response functions is also indicated in each of the tables.

DATA OBTAINED BEHIND THE SLABS

All of the measurements made behiud the slabs are summarized in Tables VIII and IX. The Bonner ball data obtained from these measurements 
are presented in Tables $X$ through XIV. Counting times and operating reactor powers for the Bonner ball measurements were sufficiently large that statistical errors in the Bonner ball counting rates may be assumed to be negligible. The reproducibility of all measurements lies within $\pm 5 \%$ and is due primarily to uncertalnties in the power calibration procedure. Backgrounds were obtained for each of the Bonner ball measurements by placing a hydrogenous slab midway between the rear face of the slabs and the detector. The data appearing in Tables X tlirough XIV have been corrected for these backgrounds. Because of the underastimation of the backgrounds in the measurement procedure described above, the data in Tables $\%$ through XIV are accurate to about $10 \%$, including uncertainties in the power calibration.

The unfolded Benjamin proton recuil spectrometer data are presented in Tables XV through XVIII, where the standard error is due to counting statistics only. The absolute energy calibration is accurate to within an estimated $\pm 5 \%$. No backgrounds were obtained for the Benjamin counter measurements because they were 1ess than $5 \%$ of the measured foregruunds.

In the region of overlap between the various Benjamin counters, no particular counter should be better than the other, and the discrepancy In the two measurements is an indication of the accuracy of the Benjamin counter system.

The unfolded NE-213 liquid scintillator spectral data are presented in l'ables XIX through XXIV, where the upper and lower limits of each unfolded spectrum are due to combined statistical and unfolding uncertainties. Backgrounds were obtained for each of the NE-213 measurements and the data in Tables XIX through XXIV are the results of the measurements after these backgrounds have been subtracted.

METHODS OF CALCULATION

The collimator geometry should be included in the calculations for all slab thicknesses, in order to take into account the effect of multiple 
reflection between the slab and the collimator, including the Iron collar, on the fluxes transmitted through the slab. Referring to Fig. 1, the composition of the iron collar may be assumed to be the same as that of the iron slabs presented in Table I. Besides the water, the only remaining collimator material that needs to be considered is a $\frac{1}{4}$-in. thickness of aluminum that contains the water that was inserted into the 14-7/8-in. diam collimator. The geometry from the location of the lead shutter inward to the reactor in Fig. I may be.ignored. The collimator is thus of cylindrical geometry and the $5 \mathrm{ft}$ by $5 \mathrm{ft}$ slabs may be assumed cylinders of $\sqrt{1.00 / \pi}=5.64 \mathrm{ft}$ diameter with negligible error. Thus, the calculations may be made using two-dimensional, $r, z$ geometry.

The calculations are best done by Monte Carlo techniques employing "point" cross sections. If groups are employed instead, a sufficient number must be used to include the most important features of the total crosssection structure in iron, which for the ORNL calculations amounted to the 220-group structure appearing in Table I.I. The cross sections are to be weighted within a group by $1 / E \Sigma_{\mathrm{T}}$. Using the multigroup. Monte Carlo method, the calculations for each slab thickness can be broken up into three parts. The first calculation replaces the collimator by a vacuum and uses as the source the absolute spectrum appearing in Table II multiplied by $212.5 \mathrm{~cm}^{2}$. The source point is sampled spatially on the exit plane of the collimator over a distance of $6.5 \mathrm{in.} \mathrm{by} \mathrm{first} \mathrm{choosing} \mathrm{an} \mathrm{incident}$ direction and then calculating the intersection of the ray with the exit plane of the collimator. Uncollided contributions to detectors located along the centerline should be calculated analytically using "point" cross sections in a separate computation using $\phi_{\text {unc }}\left(Z, E_{g}\right)=\phi_{O}\left(E_{g}\right) \times(68 / 68+Z)^{2} \int_{E_{g l}}^{E} \exp \left(-\Sigma_{T}[E] T\right) d E /\left(E_{g u}-E_{g l}\right)$,

where the $\Sigma_{T}(E)$ are "point" values in $\mathrm{cm}^{-1}$, the $\phi_{0}\left(E_{g}\right)$ are taken directly from Table II, $E_{g u}$ and $E_{g l}$ are the upper and lower limits of the group $E_{g}, T$ is the thickness of the slab in $\mathrm{cm}$, and $Z$ is the distance from the detector to the open end of the collimator, in inches. The second calculation includes the collimator and uses the same source as before, but 
but uses a coarser group structure to calculate the absolute spectral and spatial distribution of the multiply reflected current incident upon the slab over the entire exit plane of the collimator, including the iron collar. The third calculation uses the absolute source computed in the second calculation, with oimplifylng assumptions regarding the re-incident angular distribution (i.e., cosine) and spectrum, to calculate the transmitted fluxes through the slabs arising from the re-incident neutrons again using a 220-group structure. The second calculation quickly saturates with increasing slab thickness, and need not be done every time. However, the first and third calculations should be performed for every slab thickness. Biasing in the first and third steps of the calculation is optional (path length stretching of the order of a factor of two through the 24-in. and 36-in. slabs), and is not at all necessary in the second step.

The approximate contribution of the multiple-reflected collimator fluxes in this experiment is shown in Table XXV, where the calculated ratios of the transmitted fluxes above thermal energy that arise as a result of scattering in the slabs including the first, second, and third steps in the calculation to only the first step of the calculation are shown.

'l'he effect of the group structure on the transmitted spectral fluxes is shown in Table XXVI, where the results of two ANISN calculations are presented for the number of neutrons in various energy groups leaking a 1 meter radius "iron" (composition given in Table I) sphere per source fission neutron located at the center. Both cross-section sets were weighted $1 / E \sigma_{\mathrm{T}}{ }^{\prime}$.

From Table XXVI, it 1s obvious that the 220-group structure, which is tailored to the minima in the total cross section, in general produces higher fluxes leaking the sphere than the standard 100 GAM-II group structure. Hence, using such a tailored set is recommended if the ENDF/B evaluation is to be tested, with at least a $\mathrm{P}_{3}$ expansion in the angular distribution of scattering. 
Calculations of this experiment may also use the discrete ordinates technique. The collimator geometry should be included, and the virtual point anisotropic source description used. Thus the source has an intensity of $4 \pi(68 \times 2.54)^{2}=3.75 \times 10^{5}$ times the entries in Table II in neutrons/min/watt, at a point 68 in. inside the collimator, constant over the solid angle $1 \leq \cos \theta \leq 0.99887,0 \leq \phi \leq 2 \pi$, and zero elsewhere: A first collision source routine in the two-dimensional discrete ordinates calculation is recommended for the centerline detector locations, else the uncollided contribution is incorrectly calculated and difficult to extract. The uncollided contribution is calculated in a separate computation completely identical to the calculation described earlier following the Monte Carlo discussion.

A routine is also necessary to calculate the slab scattered fiuxes at the detector locations from discrete ordinate calculated fluxes in the slabs. This is done by such codes as SPACETRAN or FALSTF that are avail$a b l e$ at ORNL. The use of DOT-III, an updated version of the DOT twodimensional discrete ordinates code, is to be used, since it incorporates the first collision source and provides a tape suitable for FALSTF. The fluxes are very sensitive to the detector location, especially in the vicinity of the rear face of the slab, and the'routine SPACETRAN or FALSTF should always be used.

No therma1-neutron calculations are necessary for this experiment since the detectors used have zero sensitivity to thermal neutrons.

Calculations of the NE-213 and Benjamin counter spectra should be smoothed with the resolution function of the spectrometer before comparing with experimental data. Air attenuation from the rear face of the slabs to the detectors is to be neglected, since it was also neglected in the derivation of the source terms. Calculation of the Bonner ball counting rates involve evaluation of the following expression:

where

$$
\text { Counts/min/watt }(r)=\sum_{g} \phi\left(E_{g}, r-\Delta\right) R\left(E_{g}\right),
$$


$r$ is the distance of the geometric center of the ball from the center of the exit face of the slabs,

$\Delta$ is the center of detection correction given in Table IV, $r-\Delta$ is the location of the detector for the calculated fluxes, $R\left(E_{g}\right)$ is the interpolated response function of the Bonner ball for group $\mathrm{E}_{\mathrm{g}}$.

Note that the numbering of the groups in the response function tabulation in Tables V-VTT has been reversed oo that $R\left(E_{g}\right)$ in $E \dot{q}$. (2) appears as $R\left(E_{101-g}\right)$ in the tables. Neglect of $\wedge$ in Eq. (2) can lead to underestimates of the order of $5 \%$ in the calculated counting rates, the error being the greatest for the $10 \mathrm{in.} \mathrm{Bonner} \mathrm{ball} \mathrm{at} \mathrm{the} \mathrm{closest} \mathrm{location}$ behind the slabs. 
Table I. Composition of the Slabs in Atoms/barn-cm

\begin{tabular}{lcc}
\hline & Iron Slabs & Stainiess Steel Slabs \\
\hline Carbon & $9.815(-4)^{\mathrm{a}}$ & - \\
Manganese & $5.15(-4)$ & $1.14(-3)$ \\
Iron & $8.372(-2)$ & $5.995(-2)$ \\
Chromium & - & $1.686(-2)$ \\
Nickel & - & $7.90(-3)$ \\
\hline
\end{tabular}

$a_{\text {Read }}: 9.815 \times 10^{-4}$. 
Table II. Source Spectrum at the End of the Collimator in neuts $/ \mathrm{cm}^{2} / \mathrm{min} /$ watt $/$ group*

\begin{tabular}{|c|c|c|c|c|c|}
\hline Group & Energy Interval & Intensity & Group & Energy Interval & Intensity \\
\hline 1 & $8-10 \mathrm{MeV}$ & 190 & 31 & $1.405-1.411 \mathrm{MeV}$ & 23.8 \\
\hline 2 & $6-8$ & 665 & 32 & $1.401-1.405$ & 16.6 \\
\hline 3 & $5-6$ & 840 & 33 & $1.392-1.401$ & 35.4 \\
\hline 4 & $4-5$ & 1426 & 34 & $1.382-1.392$ & 40.1 \\
\hline 5 & $3-4$ & $2 \perp 86$ & 35 & $1.363-1.382$ & 74.5 \\
\hline 6 & $2.59-3$ & 1419 & 36 & $1.339-1.363$ & 93.5 \\
\hline 7 & $2.38-2.59$ & 887 & 37 & $1.313-1.339$ & 98.2 \\
\hline 8 & $c^{\prime} \cdot 35-2.38$ & $130^{\circ}$ & 38 & $1.306-1.313$ & 25.9 \\
\hline 9 & $2.262-2.35$ & 384 & 39 & $1.291-1.306$ & 56.8 \\
\hline 10 & $2.232-2.262$ & 130 & 40 & $1.285-1.291$ & 22.5 \\
\hline 11 & $1.943-2.232$ & 1267 & 41 & $1.251-1.285$ & 12.5 \\
\hline 12 & $1.90-1.943$ & 190 & 42 & $1.244-1.251$ & 25.9 \\
\hline 13 & $1.889-1.90$ & 47.5 & 43 & $1.221-1.244$ & 84.5 \\
\hline 14 & $1.82-1.889$ & 301 & 44 & $1.217-1.221$ & 14.2 \\
\hline 15 & $1.81-1.82$ & 43.8 & 45 & $1.211-1.217$ & $21 \cdot 3$ \\
\hline 16 & $1.783-1.81$ & 118 & 46 & $1.205-1.211$ & 21.2 \\
\hline 17 & $1.7^{1} \cdot 7-1.783$ & 153 & 47 & $1.197-1.205$ & 28.4 \\
\hline 18 & $1.722-1.747$ & 109 & 48 & $1.192-1.197$ & 17.7 \\
\hline 19 & $1.686-1.722$ & 153 & 49 & $1.169-1.192$ & 79.2 \\
\hline 20 & $1.680-1.686$ & 25.9 & 50 & $1.155-1.169$ & 48.6 \\
\hline 21 & $1.6465,-1.680$ & 143 & 51 & $1.136 \cdots 1.155$ & 65.0 \\
\hline 22 & $1.638-1.6465$ & 39.1 & 52 & $1.130 \cdots 1.136$ & 20.1 \\
\hline 23 & $1.587-1.638$ & 219 & 53 & $1.1195-1.130$ & 34.3 \\
\hline 24 & $1.567-1.587$ & 85.0 & 54 & $1.1165-1.7195$ & 10.0 \\
\hline 25 & $1.522-1.567$ & 190 & 55 & $1.1135-1.1165$ & 10.0 \\
\hline 26 & $1.506-1.522$ & 67.6 & 56 & $1.10 \%-1.1135$ & 23.8 \\
\hline 27 & $1.497-1.506$ & 38.0 & 57 & $1.098-1.107$ & 29.6 \\
\hline 28 & $1.472-1.497$ & 104 & 58 & $1.090-1.098$ & 26.0 \\
\hline 29 & $1.442-1.472$ & 125 & 59 & $1.084-1.090$ & 20.1 \\
\hline 30 & $1.411-1.442$ & 124 & 60 & $1.029-1.084$ & 177 \\
\hline
\end{tabular}


Table II (Cont'd.)

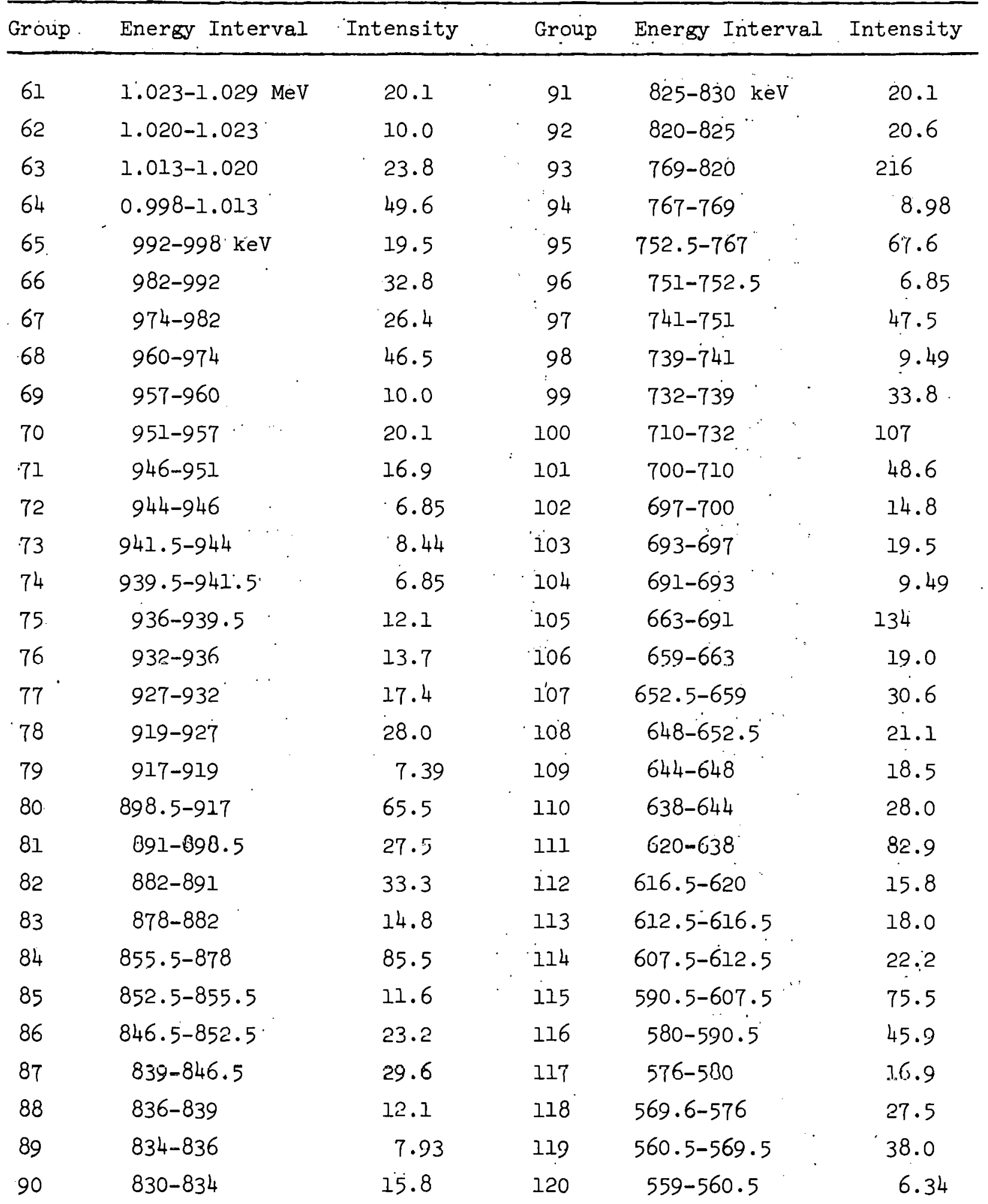


Table II (Cont'd.)

\begin{tabular}{|c|c|c|c|c|c|}
\hline Group & Energy Interval & Intensity & Group & Energy Interval & Intensity \\
\hline 121 & $557.5-559 \mathrm{keV}$ & 6.34 & 151 & $330.7-331.4 \mathrm{keV}$ & 3.66 \\
\hline 122 & $552.5-557.5$ & 21.1 & 152 & $314-330.7$ & 87.3 \\
\hline 123 & $546.5-552.5$ & 24.8 & 153 & $309 \cdot 5-314$ & 23.7 \\
\hline 124 & $543-546.5$ & 14.2 & 154 & $300-309 \cdot 5$ & 50.2 \\
\hline 125 & $540-543$ & 12.7 & 155 & $275-300$ & 135 \\
\hline $126^{\circ}$ & $536-540$ & 16.4 & 156 & $271-275$ & 21.9 \\
\hline 127 & $534-536$ & 0.7 .2 & 157 & $26 \%-271$ & 21.9 \\
\hline 128 & $515-534$ & 77.1 & 158 & $262-267$ & $27 \cdot 5$ \\
\hline 129 & $510.5-515$ & 18.0 & (y & $244.8-262$ & 94.7 \\
\hline 130 & $503-510.5$ & 29.6 & 160 & $244-244.8$ & 4.41 \\
\hline 137. & $498-503$ & $19 \cdot 5$ & 161 & $243.2-244$ & 4.41 \\
\hline 132 & $493-498$ & 19.0 & 162 & $232-243.2$ & 6.8 \\
\hline 133 & $469.1-493$ & 90.6 & 163 & $219.8-232$ & 65.5 \\
\hline 134 & $467.5-469.1$ & 5.99 & 164 & $218.6-219.8$ & $6: 34$ \\
\hline 1.35 & $464-467.5$ & 13.2 & 165 & $208-218.6$ & 56.0 \\
\hline 136 & $431.1-464$ & 99.2 & 166 & $200=208$ & 42.2 \\
\hline 137 & $436 \cdot 5-437.7$ & 4.52 & 167 & $185.2-200$ & 78.2 \\
\hline 1.28 & $433-436.5$ & 13.5 & 168 & $182-185.2$ & 16.8 \\
\hline 139 & $378-433$ & 229 & 169 & $1.75-182$ & 36.4 \\
\hline 140 & $377 \cdot 2-378$ & 3.73 & 170 & $168 \cdot 5-175$ & 34.5 \\
\hline 141 & $375-377.2$ & 10.2 & 171 & $167 \cdot 5-168.5$ & 5.38 \\
\hline 142 & $373 \cdot 5-375$ & 6.98 & 172 & $164-167.5$ & 18.9 \\
\hline 143 & $359 \cdot 3-373.5$ & 68.3 & 173 & $155-164$ & 49.6 \\
\hline 144 & $358.8-359.3$ & 2.42 & 174 & $\cdot 144-155$ & 63.9 \\
\hline 145 & $357.8-358.8$ & 4.81 & 175 & $139.5-1.44$ & 27.4 \\
\hline 146 & $357.5-357.8$ & 2.39 & 176 & $138 \cdot 3-139 \cdot 5$ & 7.45 \\
\hline 147 & $354 \cdot 5-357 \cdot 3$ & 13.8 & 177 & $136.2-138.3$ & 13.3 \\
\hline 148 & $250 \cdot 5-354 \cdot 5$ & 20.1 & 178 & $134-136.2$ & 14.0 \\
\hline 149 & $348.2-350.5$ & 11.5 & 179 & $130-134$ & 26.1 \\
\hline 150 & $331.4-348.2$. & 85.4 & 180 & $129 \cdot 2-130$ & 5.29 \\
\hline
\end{tabular}


Table II (Cont'd.)

\begin{tabular}{|c|c|c|c|c|c|c|}
\hline Group & Energy Inter & cval & Intensity & Group & Energy. Interval & Intensity \\
\hline 181 & $127.5-129.2$ & $\mathrm{keV}$ & 11.3 & 203 & $15-19 \mathrm{keV}$ & 158 \\
\hline 182 & $110-127.5$ & & 127 & 204 & $10-15$ & 264 \\
\hline 183 & $87-110$ & & 193 & 205 & $8.8-10$ & $81 \cdot 3$ \\
\hline 184 & $83-87$ & & 37.1 & 206 & $8.2-8.8$ & 44.3 \\
\hline 185 & $82.7-83$ & & 2.85 & 207 & $6.7-8.2$ & 126 \\
\hline 186 & $82.4-82.7$ & & 2.85 & 208 & $5.8-6.7$ & 87.6 \\
\hline 187 & $81.4-82.4$ & & 9.49 & 209 & $4.3-5.8$ & 174 \\
\hline 188 & $80-81.4$ & & $13 \cdot 3$ & 210 & $3 \cdot 7-4 \cdot 3$ & 86.6 \\
\hline 189 & $77-80$ & & 29.2 & $211:$ & $1.16-3.7$ & 640 \\
\hline 190 & $72-77$ & & 51.0 & 212 & $1.14-1.16$ & 8.98 \\
\hline 191 & $68-72$ & & 44.1 & 213 & $0.3167-1.14$ & 616 \\
\hline 192. & $62-68$ & & 72.4 & 214 & $88-316.7 \mathrm{eV}$ & 565 \\
\hline 193 & $38-62$ & & 354 & 215 & $24.4-88$ & 554 \\
\hline 194 & $31-38$ & & 143 & 216 & $6.79-24.4$ & 554 \\
\hline 195 & $27-31$ & & $97: 4$ & 217 & $1.89-6.79$ & 555 \\
\hline 196 & $25 \cdot 7-27$ & & 34.3 & 218 & $0.524-1.89$ & 576 \\
\hline 197 & $25.2-25 \cdot 7$ & & 13.7 & 219 & $0.145-0.524$ & 1542 \\
\hline 198 & $24.5-25.2$ & & 19.9 & $\underline{220}$ & $0-0.145$ & 6580 \\
\hline 199 & $23 \cdot 75-24 \cdot 5$ & & $21 \cdot 5$ & \multirow{2}{*}{$\begin{array}{c}\text { Totals, } \\
1-220 \\
\end{array}$} & \multirow[b]{2}{*}{$0-10 \mathrm{MeV}$} & \multirow[b]{2}{*}{$3.186 \times 10^{4}$} \\
\hline 200 & $23.25-23.75$ & & 14.8 & & & \\
\hline 201 & $22-23.25$ & & 38.3 & & . & \\
\hline 202 & $19-22$ & & 100 & & & \\
\hline
\end{tabular}

\footnotetext{
*For other group structures, interpolation in this table should follow the rule that Intensity/ln $\left[\mathrm{E}_{\mathrm{u}} / \mathrm{E}_{\ell}\right]$ is constant within the interval $\Delta E=E_{u}-E_{\ell}$, where $\Delta E$ is the tabulated energy interval.
} 
Table III. Energy Resolution of the NE-213 Spectrometer System ${ }^{\dagger}$

\begin{tabular}{|c|c|c|c|c|c|}
\hline & $a(E)$ & & $a(E)$ & & $a(E)$ \\
\hline$E(\mathrm{MeV})$ & FWHM $(\%)$ & $\mathrm{E}(\mathrm{MeV})$ & FWHM $(\%)$ & $E(\mathrm{MeV})$ & FWHM $(\%)$ \\
\hline 0.5 & 47.5 & 3.5 & 18.2 & 7.0 & 12.6 \\
\hline 0.6 & 44 & 3.6 & 18.0 & 7.2 & 12.4 \\
\hline 0.7 & 41 & 3.7 & $17 \cdot 7$ & 7.4 & 12.2 \\
\hline 0.8 & 38.5 & 3.8 & 17.4 & 7.6 & 12.1 \\
\hline 0.9 & 36 & 3.9 & 17.1 & 7.8 & 11.9 \\
\hline 1.0 & 33.5 & 4.0 & 16.9 & 8.0 & 11.8 \\
\hline $1 . \overline{1}$ & 32.5 & 4.7 & 16.7 & 8.2 & 11.6 \\
\hline 1.2 & 31 & 4.2 & .16 .5 & 8.4 & 11.5 \\
\hline 1.3 & 30 & 4.3 & 16.3 & 8.6 & 11.4 \\
\hline 1.4 & 29 & 4.4 & 16.1 & 8.8 & 11.3 \\
\hline 1.5 & $27 \cdot 5$ & 4.5 & 15.9 & 9.0 & 11.2 \\
\hline 1.6 & 26.5 & 4.6 & 15.7 & 9.2 & 11.1 \\
\hline 1.7 & 26 & 4.7 & 15.5 & 9.4 & 10.9 \\
\hline 1.8 & 25 & 4.8 & 15.3 & 9.6 & 10.8 \\
\hline 1.9 & 24.5 & 4.9 & 15.2 & 9.8 & 10.7 \\
\hline 2.0 & 24 & 5.0 & 15.1 & 10.0 & 10.5 \\
\hline 2.1 & 23.5 & 5.1 & 14.9 & 10.2 & 10.3 \\
\hline 2.2 & 23 & 5.2 & 14.7 & 10.4 & 10.2 \\
\hline 2.3 & 22.5 & 5.3 & 14.5 & 10.6 & 10.1 \\
\hline 2.4 & 22 & 5.4 & 14.4 & 10.8 & 10.0 \\
\hline 2.5 & 21.5 & 5.5 & 14.3 & 11.0 & 9.8 \\
\hline 2.6 & 21.2 & 5.6 & 14.2 & 11.4 & 9.7 \\
\hline 2.7 & 20.0 & 5.7 & 14.1 & 11.8 & 9.6 \\
\hline 2.8 & 20.4 & 5.8 & 13.9 & 12.2 & 9.6 \\
\hline 2.9 & 20.1 & 5.9 & 13.8 & & \\
\hline 3.0 & 19.7 & 6.0 & 13.7 & & \\
\hline 3.1 & 19.4 & 6.2 & 13.5 & & \\
\hline 3.2 & 19.1 & 6.4 & 13.2 & & \\
\hline 3.3 & 18.8 & 6.6 & 13.0 & & \\
\hline 3.4 & 18.5 & 6.8 & 12.8 & & \\
\hline
\end{tabular}

Interpolation in this table should follow the formula'

$$
a(E)=\frac{E_{2}-E}{E_{2}-E_{1}} a\left(E_{1}\right)+\frac{E-E_{1}}{E_{2}-E_{1}} a\left(E_{2}\right) \text {, where } E_{1} \leq E \leq E_{2} \text {. }
$$


Table IV. Bonner Ball Description

Spherical, 2-in.-diam ${ }^{10} \mathrm{BF}_{3}$ Proportional Counter Surrounded by Polyethyiene and 0.030 in. Cadmium

\begin{tabular}{ccccc}
$\begin{array}{c}\text { Standard } \\
\text { Bonner Ball } \\
\begin{array}{c}\text { Designation } \\
\text { (in.) }\end{array}\end{array}$ & $\begin{array}{c}\text { Polyethylene } \\
\text { Thickness } \\
\text { (in.) }\end{array}$ & $\begin{array}{c}\text { Polyethylene } \\
\text { Density } \\
\text { (grams/cm })^{3}\end{array}$ & $\begin{array}{c}\text { Diameter } \\
\text { of Ball } \\
\text { (in.) }\end{array}$ & $\begin{array}{c}\text { Location of Center } \\
\text { of Detection from } \\
\text { Center of Ball a } \\
\text { (in.) }\end{array}$ \\
\hline 3 & 0.515 & 0.951 & 3.09 & 0.9 \\
6 & 1.91 & 0.925 & 5.88 & 1.8 \\
10 & 3.90 & $0.93 \perp$ & 9.86 & 3.0 \\
\hline
\end{tabular}

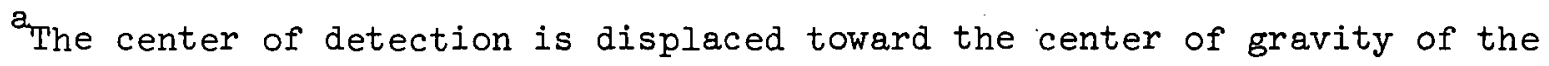
hemispherical surface upon which the neutrons are incident. 
Table V. Response for 3.09 Inch Diameter Bonner Sphere*

\begin{tabular}{|c|c|c|}
\hline $\begin{array}{l}\text { Group } \\
\text { No. }\end{array}$ & $\begin{array}{c}\text { Midpoint Energy } \\
(\mathrm{eV})\end{array}$ & $\begin{array}{c}\text { Response }{ }^{\dagger} \\
\text { (counts/incident neut } / \mathrm{cm}^{2} \text { ) }\end{array}$ \\
\hline 1 & Thermal & Negligible \\
\hline 2 & $4.73(-1)$ & $4.81(-1)$ \\
\hline 3 & $6.07(-1)$ & $8.03(-1)$ \\
\hline 4 & $7.79(-1)$ & $9.95(-1)$ \\
\hline 5 & $1.00(0)$ & $1.11(0)$ \\
\hline 6 & $1.29(0)$ & $1.17(0)$ \\
\hline 7 & $1.65(0)$ & $1.19(0)$ \\
\hline 8 & $2.12(0)$ & $1.19(0)$ \\
\hline 9 & $2.72(0)$ & $1.18(0)$ \\
\hline 10 & $3.49(0)$ & $1.16(0)$ \\
\hline 11 & $4.49(0)$ & $1.14(0)$ \\
\hline 12 & $5.76(0)$ & $1.11(0)$ \\
\hline 13 & $7.40(0)$ & $1.08(0)$ \\
\hline 14 & $9.50(0)$ & $1.05(0)$ \\
\hline 15 & $1.22(1)$ & $1.02(0)$ \\
\hline 16 & $1.57(1)$ & $9.89(-1)$ \\
\hline 17 & $2.01(1)$ & $9.55(-1)$ \\
\hline 18 & $2.58(1)$ & $8.73(-1)$ \\
\hline 19 & $3.31(1)$ & $8.87(-1)$ \\
\hline 20 & $4.26(1)$ & $8.57(-1)$ \\
\hline 21 & $5.46(1)$ & $8.25(-1)$ \\
\hline 22 & $7.01(1)$ & $7.72(-1)$ \\
\hline 23 & $9.01(1)$ & $6.78(-1)$ \\
\hline 24 & $1.16(2)$ & $8.35(-1)$ \\
\hline 25 & $1.49(2)$ & $0.69(-1)$ \\
\hline 26 & $1.91(2)$ & $8.41(-1)$. \\
\hline 27 & $2.45(2)$ & $8.02(-1)$ \\
\hline 28 & $3.14(2)$ & $7.72(-1)$ \\
\hline 29 & $4.04(2)$ & $7.43(-1)$ \\
\hline 30 & $5.18(2)$ & $T .01(-1)$ \\
\hline 31 & $6.66(2)$ & $6.82(-1)$ \\
\hline 32 & $8.55(2)$ & $6.54(-1)$ \\
\hline 33 & $1.10(3)$ & $6.25(-1)$ \\
\hline 34 & $1.41(3)$ & $6.00(-1)$ \\
\hline 35 & $1.81(3)$ & $5.73(=1)$ \\
\hline 36 & $2.32(3)$ & $5.48(-1)$ \\
\hline 37 & $2.98(3)$ & $5.25(-1)$ \\
\hline 38 & $3.83(3)$ & $5.02(-1)$ \\
\hline 39 & $4.92(3)$ & $1.81(-1)$ \\
\hline 40 & $6.32(3)$ & $4.61(-1)$ \\
\hline 41 & $8.11(3)$ & $4.41(-1)$ \\
\hline 42 & $1.04(4)$ & $4.22(-1)$ \\
\hline
\end{tabular}

*Radial thickness of polyethylene $=0.515$ inches: Density of polyethylene $=0.951 \mathrm{gram} / \mathrm{cc}$. Estimated accuracy is $\pm 10 \%$ over the entire energy range. 
Table V. (Cont!d:)

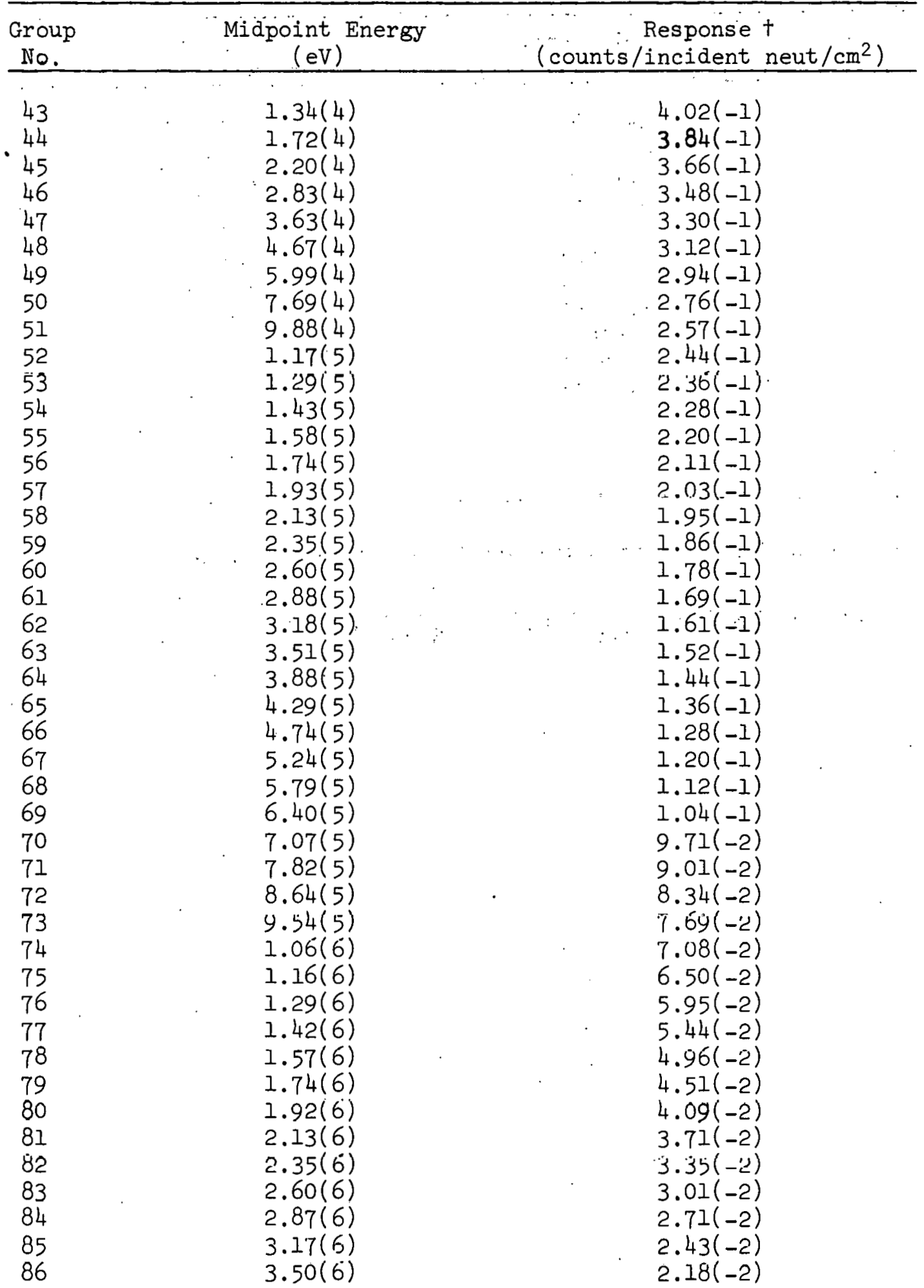


Table V (Cont'd.)

\begin{tabular}{ccc}
$\begin{array}{c}\text { Group } \\
\text { No. }\end{array}$ & $\begin{array}{c}\text { Midpoint Energy } \\
(\mathrm{eV})\end{array}$ & $\begin{array}{c}\text { Response }+ \\
\text { (counts } / \text { incident neut } / \mathrm{cm}^{2} \text { ) }\end{array}$ \\
\hline 87 & $3.87(6)$ & $1.95(-2)$ \\
88 & $4.28(6)$ & $1.73(-2)$ \\
89 & $4.73(6)$ & $1.53(-2)$ \\
90 & $5.23(6)$ & $1.48(-2)$ \\
91 & $5.78(6)$ & $1.34(-2)$ \\
92 & $6.38(6)$ & $1.19(-2)$ \\
93 & $7.06(6)$ & $9.83(-3)$ \\
94 & $7.80(6)$ & $9.03(-3)$ \\
95 & $8.62(6)$ & $7.95(-3)$ \\
96 & $9.52(6)$ & $7.12(-3)$ \\
97 & $1.05(7)$ & $6.35(-3)$ \\
98 & $1.16(7)$ & $5.57(-3)$ \\
99 & $1.29(7)$ & $4.98(-3)$ \\
100 & $1.42(7)$ & $4.40(-3)$ \\
\hline
\end{tabular}

tInterpolation in this table should follow the formula

$$
R(E)=R\left(E_{1}\right) \cdot \frac{E_{2}-E}{E_{2}-E_{1}}+R\left(E_{2}\right) \cdot \frac{E-E_{1}}{E_{2}-E_{1}} \text {, for } E_{1} \leq E \leq E_{2} \text {. }
$$


Table VI. Response for 5.88 . Inch. Diameter Bonner Sphere*

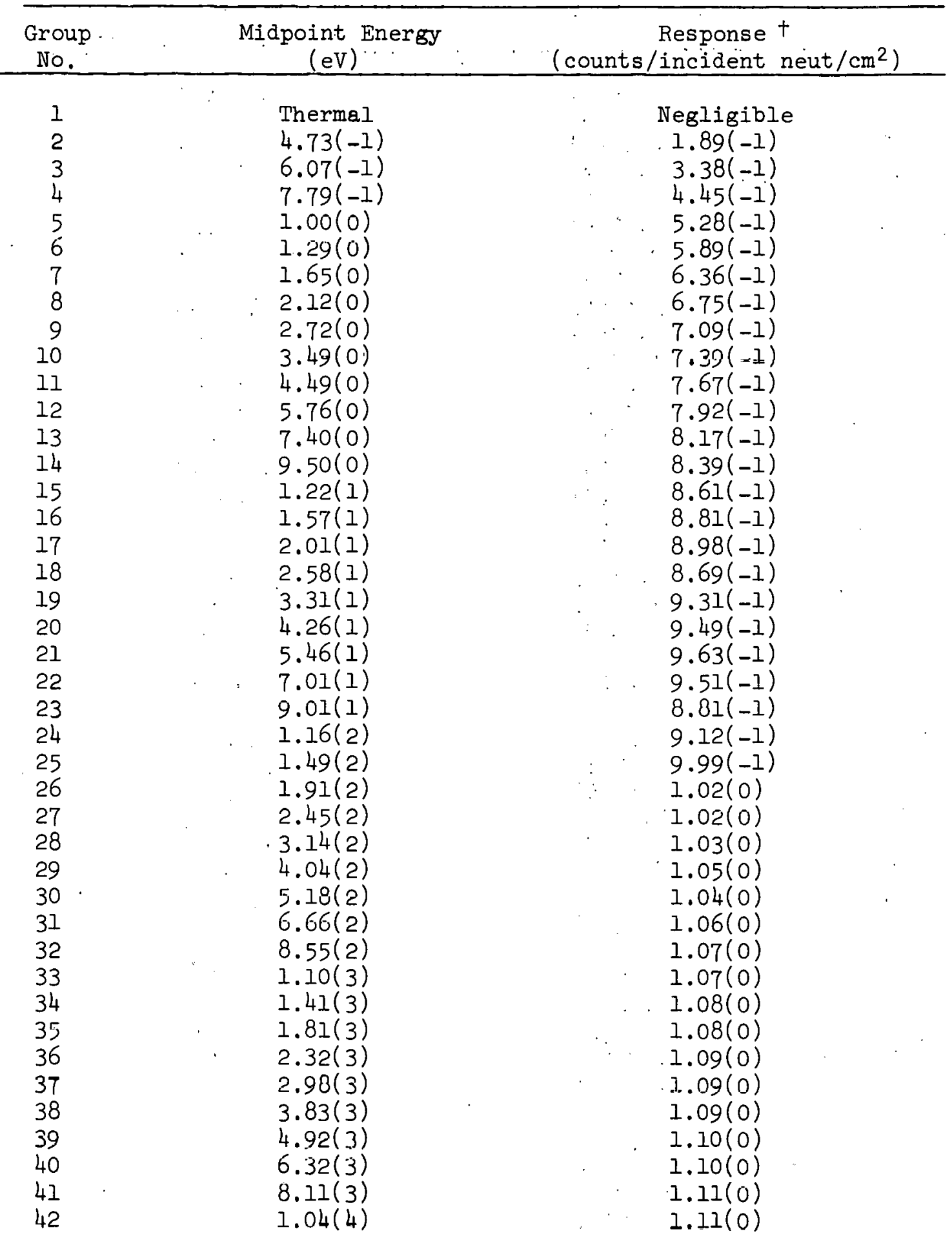

*Radial thickness of polyethylene $=1.910$ inches: density of polyathylene $=0.925 \mathrm{gram} / \mathrm{sc}$. Fst.imated accurary is $\pm 10 \%$ over the entire energy range. 
Table VI (Cont'd.)

\begin{tabular}{|c|c|c|}
\hline $\begin{array}{l}\text { Group } \\
\text { No. }\end{array}$ & $\begin{array}{l}\text { Midpoint Energy } \\
(\mathrm{eV})\end{array}$ & $\begin{array}{c}\text { Response }{ }^{\dagger} \\
\text { (counts/incident neut } / \mathrm{cm}^{2} \text { ) }\end{array}$ \\
\hline 43 & $1.34(4)$ & $1.11(0)$ \\
\hline 44 & $1.72(4)$ & $1.12(0)$ \\
\hline 45 & $2.20(4)$ & $1.13(0)$ \\
\hline 46 & $2.83(4)$ & $1.14(0)$ \\
\hline 47 & $3.63(4)$ & $1.15(0)$ \\
\hline 48 & $4.67(4)$ & $1.16(0)$ \\
\hline 49 & $5.99(4)$ & $1.18(0)$ \\
\hline bo & $7.69(4)$ & $1.20(0)$ \\
\hline 51 & $9.08(4)$ & $1.25(0)$ \\
\hline 52 & $1.17(5)$ & $1: 23(0)$ \\
\hline 53 & $1.29(5)$ & $1.24(0)$ \\
\hline 54 & $1.43(5)$ & $1.25(0)$ \\
\hline 55 & $1.58(5)$ & $1.26(0)$ \\
\hline 56 & $1.74(5)$ & $1.27(0)$ \\
\hline 57 & $1.93(5)$ & $1.28(0)$ \\
\hline 58 & $2.13(5)$ & $1.28(0)$ \\
\hline 59 & $2.35(5)$ & $1.29(0)$ \\
\hline 60 & $2.60(5)$ & $1.30(0)$ \\
\hline 61 & $2.88(5)$ & $1.39(0)$ \\
\hline 62 & $3.18(5)$ & $1.31(0)$ \\
\hline 63 & $3.51(5)$ & $1.31(0)$ \\
\hline 64 & $3.88(5)$ & $1.31(0)$ \\
\hline 63 & $4.29(5)$ & $1.31(0)$ \\
\hline 66 & $4.74(5)$ & $1.30(0)$ \\
\hline 67 & $5.24(5)$ & $1.30(0)$ \\
\hline 68 & $5.79(5)$ & $1.29(0)$ \\
\hline 69 & $6.40(5)$ & $1.28(0)$ \\
\hline 70 & $7.07(5)$ & $1.26(0)$ \\
\hline 71 & $7.82(5)$ & $1.25(0)$ \\
\hline 72 & $8.64(5)$ & $1.23(0)$ \\
\hline 73 & $9.54(5)$ & $1.20(0)$ \\
\hline 74 & $1.06(6)$ & $1.18(0)$ \\
\hline 75 & $1.16(6)$ & $1.15(0)$ \\
\hline 76 & $1.29(6)$ & $1.11(0)$ \\
\hline 77 & $1.42(6)$ & $1.08(0)$ \\
\hline 78 & $1.57(6)$ & $1.04(0)$ \\
\hline 79 & $1.74(6)$ & $9.99(-1)$ \\
\hline 80 & $1.92(6)$ & $9.56(-1)$ \\
\hline 81 & $2.13(6)$ & $9.06(-1)$ \\
\hline 82 & $2.35(6)$ & $8.65(-1)$ \\
\hline 83 & $2.60(6)$ & $8.14(-1)$ \\
\hline 84 & $2.07(6)$ & $7.55(-1)$ \\
\hline 85 & $3.17(6)$ & $7.17(-1)$ \\
\hline 86 & $3.50(6)$ & $6.57(-1)$ \\
\hline
\end{tabular}


Table VI (Cont'd..)

\begin{tabular}{lcc}
$\begin{array}{c}\text { Group } \\
\text { No. }\end{array}$ & $\begin{array}{c}\text { Midpoint Energy } \\
(\mathrm{eV})\end{array}$ & $\begin{array}{c}\text { Response }+ \\
\text { (counts/incident neut } / \mathrm{cm}^{2} \text { ) }\end{array}$ \\
\hline 8 & $3.87(6)$ & $6.20(-1)$ \\
88 & $4.28(6)$ & $5.84(-1)$ \\
89 & $4.73(6)$ & $5.39(-1)$ \\
90 & $5.23(6)$ & $5.09(-1)$ \\
91 & $5.78(6)$ & $4.81(-1)$ \\
92 & $6.38(6)$ & $4.52(-1)$ \\
93 & $7.06(6)$ & $3.96(-1)$ \\
94 & $7.80(6)$ & $3.69(-1)$ \\
95 & $8.62(6)$ & $3.28(-1)$ \\
96 & $9.52(6)$ & $2.94(-1)$ \\
97 & $1.05(7)$ & $2.62(-1)$ \\
98 & $1.16(7)$ & $2.28(-1)$ \\
99 & $1.29(7)$ & $2.02(-1)$ \\
100 & $1.42(7)$ & $1.80(-1)$ \\
\hline
\end{tabular}

tInterpolation in this table should follow the formula:

$$
R(E)=R\left(E_{1}\right) \cdot \frac{E_{2}-E}{E_{2}-E_{1}}+R\left(E_{2}\right) \cdot \frac{E-E_{1}}{E_{2}-E_{1}}, \text { for } E_{1} \leq E \leq E_{2} \text {. }
$$


Table VII. Response for 9.86 Inch Diameter Bonner Sphere*

\begin{tabular}{|c|c|c|}
\hline $\begin{array}{l}\text { Group } \\
\text { No. }\end{array}$ & $\begin{array}{c}\text { Midpoint Energy } \\
(\mathrm{eV})\end{array}$ & $\begin{array}{c}\text { Response } t \\
\text { (counts/incident neut } / \mathrm{cm}^{2} \text { ) }\end{array}$ \\
\hline 1 & Thermal & Negligible \\
\hline 2 & $4.73(-1)$ & $2.43(-2)$ \\
\hline 3 & $6.07(-1)$ & $4.36(-2)$ \\
\hline 4 & $7.79(-1)$ & $5.76(-2)$ \\
\hline 5 & $1.00(0)$ & $6.85(-2)$ \\
\hline 6 & $1.29(0)$ & $7.66(-2)$ \\
\hline 7 & $1.65(0)$ & $8.28(-2)$ \\
\hline 8 & $2.12(0)$ & $8.81(-2)$ \\
\hline 9 & $2.72(0)$ & $9.29(-2)$ \\
\hline 10 & $3.49(0)$ & $9.72(-2)$ \\
\hline 11 & $4.49(0)$ & $1.01(-1)$ \\
\hline 12 & $5.76(0)$ & $1.05(-1)$ \\
\hline 13 & $7.40(0)$ & $1.09(-1)$ \\
\hline 14 & $9.50(0)$ & $1.13(-1)$ \\
\hline 15 & $1.22(1)$ & $1.16(-1)$ \\
\hline 16 & $1.57(1)$ & $1.20(-1)$ \\
\hline 17 & $2.01(1)$ & $1.23(-1)$ \\
\hline 18 & $2.58(1)$ & $1.20(-1)$ \\
\hline 19 & $3.31(1)^{\circ}$ & $1.30(-1)$ \\
\hline 20 & $4.26(1)$ & $1.34(-1)$ \\
\hline 21 & $5.46(1)$ & $1.37(-1)$ \\
\hline 22 & $7.01(1)$ & $1.37(-1)$ \\
\hline 23 & $9.01(1)$ & $1.28(-1)$ \\
\hline 24 & $1.16(2)$ & $1.34(-7)$ \\
\hline 25 & $1.49(2)$ & $1.49(-1)$ \\
\hline $26^{\circ}$ & $1.91(2)$ & $1.53(-1)$ \\
\hline 27 & $2.45(2)$ & $1.56(-1)$ \\
\hline 28 & $3.14(2)$ & $1.60(-1)$ \\
\hline 29 & $4.04(2)$ & $1.64(-1)$ \\
\hline 30 & $5.18(2)$ & $1.65(-1)$ \\
\hline 31 & $6.66(2)$ & $1.71(.1)$ \\
\hline 32 & $8.55(2)$ & $1.75(-1)$ \\
\hline 33 & $1.10(3)$ & $1.79(-1)$ \\
\hline 34 & $1.41(3)$ & $1.83(-1)$ \\
\hline 3.5 & $1.81(3)$ & $1.86(-1)$ \\
\hline 36 & $2.32(3)$ & $1.20(-1)$ \\
\hline 31 & $2.98(3)$ & $1.94(-1)$ \\
\hline 38 & $3.83(3)$ & $1.98(-1)$ \\
\hline 39 & $4,92(3)$ & $2.02(-1)$ \\
\hline 40 & $6.32(3)$ & $2.07(-1)$ \\
\hline 41 & $8.11(3)$ & $2.12(-1)$ \\
\hline 42 & $1.04(4)$ & $2.18(-1)$ \\
\hline
\end{tabular}

* Radial Thickness of polyethylene $=4.890$ inches: density of polyethylene $=0.951 \mathrm{gram} / \mathrm{cc}$. Estimated accuracy is $+10 \%$ for energies above $1 \mathrm{keV}$ and $\pm 25 \%$ for energies below $1 \mathrm{keV}$. 
Table VII (Cont'd.)

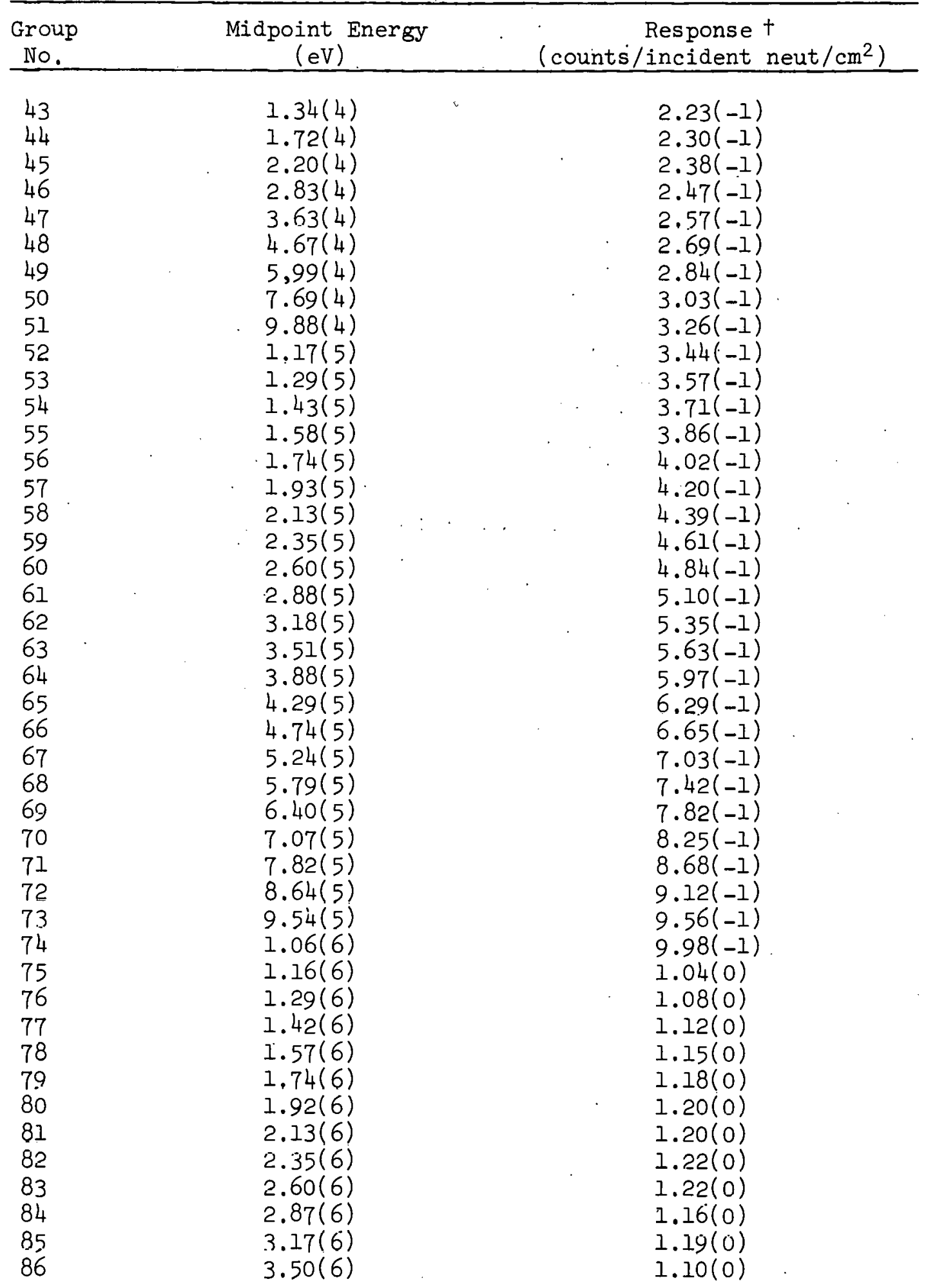


Table VII (Cont'd.)

\begin{tabular}{ccc}
\hline $\begin{array}{c}\text { Group } \\
\text { No. }\end{array}$ & $\begin{array}{c}\text { Midpoint Energy } \\
(\mathrm{eV})\end{array}$ & $\begin{array}{c}\text { Response }+ \\
\text { (counts/incident neut/cm }{ }^{2} \text { ) }\end{array}$ \\
\hline 87 & $3.87(6)$ & $1.11(0)$ \\
88 & $4.28(6)$ & $1.14(0)$ \\
89 & $4.73(6)$ & $1.12(0)$ \\
90 & $5.23(6)$ & $1.10(0)$ \\
91 & $5.78(6)$ & $1.06(0)$ \\
92 & $6.38(6)$ & $1.03(0)$ \\
93 & $7.06(6)$ & $9.87(-1)$ \\
94 & $7.80(6)$ & $9.13(-1)$ \\
95 & $8.62(6)$ & $8.714(-1)$ \\
96 & $9.52(6)$ & $8.05(-1)$ \\
97 & $1.05(7)$ & $7.40(-1)$ \\
98 & $1.16(7)$ & $6.61(-1)$ \\
99 & $1.29(7)$ & $6.05(-1)$ \\
100 & $1.42(7)$ & $5.52(-1)$ \\
\hline
\end{tabular}

tinterpolation in this table should follow the formula

$$
R(E)=R\left(E_{1}\right) \cdot \frac{E_{2}-E}{E_{2}-E_{1^{\prime}}}+R\left(E_{2}\right) \cdot \frac{E-E_{1}}{E_{2}-E_{1}} \text {, for } E_{1} \leq E \leq E_{2} \text {. }
$$


Table VIII. Experimental Configuration for the Iron Slabs

\begin{tabular}{|c|c|c|c|c|c|}
\hline \multirow[b]{2}{*}{$\begin{array}{l}\text { Nominal Iron } \\
\text { Thickness } \\
\text { (in.) } \\
\end{array}$} & \multirow[b]{2}{*}{$\begin{array}{c}\text { Actual Iron } \\
\text { Thickness } \\
\text { (in.) }\end{array}$} & \multicolumn{3}{|c|}{ Detector Locations* } & \multirow[b]{2}{*}{ Detector Type } \\
\hline & & $\begin{array}{l}\text { Centerline Distance } \\
\text { Behind Slab (in.) }\end{array}$ & $\begin{array}{l}\text { Radial Distance } \\
\text { from Centerline } \\
\text { (in.) }\end{array}$ & $\begin{array}{c}\text { Observation Angle } \\
\text { with Respect to } \mathrm{E} \\
\text { (deg) }\end{array}$ & \\
\hline 1.5 & 1.55 & $\begin{array}{l}152 \\
146 \\
107 \\
\end{array}$ & $\begin{array}{r}0 \\
39 \\
107 \\
\end{array}$ & $\begin{array}{r}0 \\
15 \\
45 \\
\end{array}$ & Bonner Ball \\
\hline 4 & 4.05 & $\begin{array}{l}158 \\
116 \\
\end{array}$ & $\begin{array}{r}42.5 \\
116 \\
\end{array}$ & $\begin{array}{l}15 \\
45 \\
\end{array}$ & $\begin{array}{l}\mathrm{NE}-213 \text { Spec- } \\
\text { trometer }\end{array}$ \\
\hline 6 & 6.06 & 162 & 0 & 0 & $\begin{array}{l}\mathrm{NE}-213 \text { Spec- } \\
\text { trometer }\end{array}$ \\
\hline \multirow[t]{3}{*}{12} & . 12.13 & $\begin{array}{l}141 \\
136 \\
100 \\
\end{array}$ & $\begin{array}{c}0 \\
36.5 \\
100 \\
\end{array}$ & $\begin{array}{r}0 \\
15 \\
45 \\
\end{array}$ & Bonner Balls \\
\hline & & $\begin{array}{l}156 \\
150 \\
110 \\
\end{array}$ & $\begin{array}{r}0 \\
40.5 \\
110 \\
\end{array}$ & $\begin{array}{r}0 \\
15 \\
45 \\
\end{array}$ & $\begin{array}{l}\text { NE-213 Spec- } \\
\text { trometer }\end{array}$ \\
\hline & 12.25 & $\begin{array}{l}10 \\
10 \\
\end{array}$ & $\begin{array}{r}0 \\
12 \\
\end{array}$ & $\begin{array}{r}0 \\
50 \\
\end{array}$ & $\begin{array}{l}\text { Benjamin } \\
\text { Spectrometer }\end{array}$ \\
\hline 24 & 24.41 & $\begin{array}{r}128 \\
124 \\
90.5 \\
\end{array}$ & $\begin{array}{c}0 \\
33 \\
90.5 \\
\end{array}$ & $\begin{array}{r}0 \\
15 \\
45 \\
\end{array}$ & Bonner Balls \\
\hline 36 & 36.56 & $\begin{array}{l}115 \\
111 \\
81.5\end{array}$ & $\begin{array}{c}0 \\
30 \\
81.5\end{array}$ & $\begin{array}{r}0 \\
15 \\
45\end{array}$ & Bonner Balls \\
\hline
\end{tabular}

*Distances are measured to the gemetric center of the Bonner balls, and to the center of detection for the other detectors.

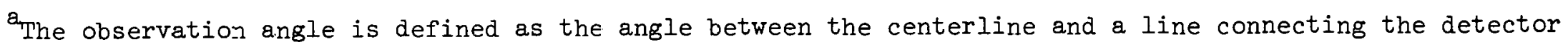
with the midpoint of the emergent face of the slab. The vertex of this angle is the pivot point for the angular traverses. 
Table IX. Experimental Configurations for the Stainless Steel Slab

\begin{tabular}{|c|c|c|c|c|c|}
\hline \multirow[b]{2}{*}{$\begin{array}{l}\text { Nominal SS } \\
\text { Thickness } \\
\text { (in.) }\end{array}$} & \multirow[b]{2}{*}{$\begin{array}{l}\text { Actual SS } \\
\text { Thickness } \\
\text { (in.) }\end{array}$} & \multicolumn{3}{|c|}{ Detector Locations ${ }^{*}$} & \multirow[b]{2}{*}{ Detector Type } \\
\hline & & $\begin{array}{c}\text { Centerline Distance } \\
\text { Eehind Slab } \\
\text { (in.) }\end{array}$ & $\begin{array}{c}\text { Radial Distance } \\
\text { fron Centerline } \\
\text { (in.) }\end{array}$ & $\begin{array}{c}\text { Observatian Angle } \\
\text { with Pespect To } \\
\text { (deg) }\end{array}$ & \\
\hline \multirow[t]{5}{*}{12} & 12.17 & 141 & 0 & 0 & Bonner Balls \\
\hline & & 135 & 36.5 & 15 & \\
\hline & & 100 & 100 & 45 & \\
\hline & & 10 & 0 & J & Benjamin \\
\hline & & 10 & 12 & 53 & Spectrometer \\
\hline
\end{tabular}

*Distances are measured to the geometric center of the Bonner balls, and tc the center of detection for the other detectors.

$a_{T h e}$ observation angle is defired as the angle between the centerline and a ine connecting the detector with the midpoint of the emergent face of the slab. The vertex of this angie is the pivot point for the angular traverses. 
Table X. Bonner Ball Counting Rates Behind 1.5 in. of Iron (cts/min/watt)

\begin{tabular}{cccc}
\hline Bonner Ball & 3-in. & 6-in. & $\frac{10-\text { in. }}{$\cline { 3 - 4 } \text {$On centerline }}$ \\
\cline { 2 - 4 } $15^{\circ}$ & 57.8 & 607 & 587 \\
$45^{\circ}$ & 1.02 & 5.22 & 4.74 \\
\hline
\end{tabular}

Table XI. Bonner Ball Counting Rates Behind 12 in. of Iron (cts/min/watt)

\begin{tabular}{cccc}
\hline Bonner Ball & 3-in. & $\frac{6-i n .}{10-i n .}$ \\
\cline { 2 - 4 } On centerline & 2.32 & 18.5 & $\frac{1.89}{15^{\circ}}$ \\
$45^{\circ}$ & 0.577 & 3.54 & 1.58 \\
\hline
\end{tabular}

Table XII. Bonner Ball Counting Rates Behind 24 in. of Iron (cts/min/watt)

\begin{tabular}{cccc}
\hline Bonner Ball & 3-in. & $\frac{6-i n .}{3.49}$ & $\frac{10-i n}{1.40}$ \\
On centerline & 0.604 & 1.70 & 0.600 \\
$15^{\circ}$ & 0.367 & 1.13 & 0.405 \\
$45^{\circ}$ & 0.245 & \\
\hline
\end{tabular}

Table XIII. Bonner Bail Counting Rates Behind 36 in. of Iron (cts/min/watt)

\begin{tabular}{cccc}
\hline Bonner Ball & 3-in. & $\frac{6-i n .}{10-i n}$ \\
\cline { 2 - 2 } On Centerline & 0.243 & 1.08 & $\frac{0.368}{15^{\circ}}$ \\
$45^{\circ}$ & 0.181 & 0.700 & 0.227 \\
\hline
\end{tabular}


Table XIV. Bonner Ball Counting Rates Behind 12 in. of Stainless Steel (cts/min/watt)

\begin{tabular}{llll}
\hline Bonner Ball & 3-in. & 6-in. & 10-in. \\
\hline On Centerline & 0.783 & 5.97 & 3.51 \\
$15^{\circ}$ & 0.496 & 2.74 & 1.28 \\
$45^{\circ}$ & 0.327 & 1.77 & 0.797 \\
\hline
\end{tabular}


Table XV. Benjamin Counter Spectrum on the Centerline 10 in. Behind $12 \mathrm{in.}$ of Iron

\begin{tabular}{|c|c|c|}
\hline $\begin{array}{c}\text { Energy Interval } \\
(\mathrm{keV})\end{array}$ & $\begin{array}{c}\text { Flux } \\
\text { (neuts } / \mathrm{cm}^{2} / \mathrm{MeV} / \mathrm{min} / \text { watt) }\end{array}$ & $\begin{array}{l}\text { Standard } \\
\text { Error }(\%)\end{array}$ \\
\hline \multicolumn{3}{|c|}{10 Atmosphere Counter } \\
\hline $1379 \cdot 3-1500$ & 20.4 & 9.6 \\
\hline $1277.1-1379 \cdot 3$ & $32 \cdot 3$ & 7.4 \\
\hline $1184.2-1277.1$ & 46.4 & 5.8 \\
\hline $1091.3-1184.2$ & 52.4 & 5.2 \\
\hline $1017.0-1091.3$ & 52.4 & 6.7 \\
\hline $933.4-1017.0$ & 65.7 & 4.6 \\
\hline $868.4-933.4$ & 59.2 & 6.6 \\
\hline $803.4-868.4$ & 54.8 & 6.8 \\
\hline $738.4-803.4$ & 91.6 & 3.9 \\
\hline $682.7-738.4$ & 158 & 2.7 \\
\hline $636.2-682.7$ & 195 & 2.7 \\
\hline $589.8-636.2$ & 182 & 2.9 \\
\hline \multicolumn{2}{|r|}{3 Atmosphere Counter } & \\
\hline $644 \cdot 7-700$ & 228 & 3.5 \\
\hline $597.9-644.7$ & 233 & 4.2 \\
\hline $551.1-597.9$ & 133 & 7.0 \\
\hline $512.8-551.1$ & 108 & 10.4 \\
\hline $474.5-512.8$ & 118 & 8.8 \\
\hline $436.2-474.5$ & 98.7 & 9.7 \\
\hline $406.4-436.2$ & 129 & 9.2 \\
\hline $372 \cdot 3-406.4$ & 233 & 4.1 \\
\hline $346.8-372.3$ & 309 & 4.1 \\
\hline $321.3-346.8$ & 351 & 3.5 \\
\hline $295 \cdot 7-321 \cdot 3$ & 372 & 3.1 \\
\hline $274.5-295.7$ & 340 & 4.0 \\
\hline $253.2-274.5$ & 254 & 5.0 \\
\hline $236.2-253.2$ & 220 & 6.8 \\
\hline $214.9-236.2$ & 180 & 6.0 \\
\hline
\end{tabular}


Table XV (Cont:d.)

\begin{tabular}{ccc}
$\begin{array}{c}\text { Energy Interval } \\
(\mathrm{keV})\end{array}$ & $\begin{array}{c}\text { Flux } \\
\text { (neuts } / \mathrm{cm}^{2} / \mathrm{MeV} / \mathrm{min} / \text { watt) }\end{array}$ & $\begin{array}{c}\text { Standard } \\
\text { Error }(\%)\end{array}$ \\
\hline I Atmosphere Counter & \\
$275.5-300$ & 380 & 4.0 \\
$255.4-275.5$ & 291 & 6.3 \\
$237.5-255.4$ & 215 & 9.2 \\
$219.7-237.5$ & 204 & 8.9 \\
$201.9 \cdots 219.7$ & 179 & 9.2 \\
$186.2-201.9$ & 273 & 6.5 \\
$172.9-186.2$ & 384 & 5.1 \\
$159.5-172.9$ & 312 & 5.9 \\
$148.3-159.5$ & 334 & 6.2 \\
$137.2-148.3$ & 592 & 3.3 \\
$126.0-137.2$ & 528 & 3.5 \\
$117.1-126.0$ & 317 & 6.8 \\
$108.2-117.1$ & 192 & 10.3 \\
$99.3-108.2$ & 129 & 14.0 \\
$92.6-99.3$ & 156 & 14.0 \\
$85.9-92.6$ & 222 & 9.1 \\
& &
\end{tabular}


Table XVI. Benjamin Counter Spectrum 12 in. off the Centerline and $10^{\prime \prime}$ in. Behind 12 in. of Iron

\begin{tabular}{|c|c|c|}
\hline $\begin{array}{c}\text { Energy Interval } \\
(\mathrm{keV})\end{array}$ & $\begin{array}{c}\mathrm{Flux} \\
\text { (neuts } / \mathrm{cm}^{2} / \mathrm{MeV} / \mathrm{min} / \text { watt) }\end{array}$ & $\begin{array}{l}\text { Standard } \\
\text { Error }(\%)\end{array}$ \\
\hline & \multicolumn{2}{|l|}{10 Atmosphere Counter } \\
\hline $1379 \cdot 3-1500$ & 4.97 & 12.0 \\
\hline $1277.1-1379.3$ & 6.81 & 10.6 \\
\hline $1184.2-1277.1$ & 10.3 & 7.9 \\
\hline $1091.3-1184.2$ & 12.4 & $: 6.6$ \\
\hline $1017: 0-1091.3$ & 14.7 & $.7 \cdot 4$ \\
\hline $933.4-1017.0$ & 18.6 & 5.0 \\
\hline $868.4-933.4$ & 21.4 & $5.9^{\circ}$ \\
\hline $803.4-868.4$ & 20.7 & 6.0 \\
\hline $738.4-803.4$ & $20 \cdot 7$ & 6.0 \\
\hline $738.4-803.4$ & $31 \cdot 3$ & 3.9 \\
\hline $682.7-738.4$ & .52 .7 & 2.8 \\
\hline $636.2-682.7$ & 74.8 & $2 \cdot 5$ \\
\hline \multirow[t]{2}{*}{$589.8-636.2$} & 72.4 & 2.6 \\
\hline & 3 Atmosphere Counter & . \\
\hline $645.0-700$ & 69.2 & 4.4 \\
\hline $598.5-645.0$ & 95.8 & 4.0 \\
\hline $552.0-598.5$ & 70.0 & 5.5 \\
\hline $509.7-552.0$ & $54 \cdot 5$ & 7.6 \\
\hline $471.6-509.7$ & 60.8 & $7 \cdot 3$ \\
\hline $437.8-471.6$ & 54.3 & 8.8 \\
\hline $403.9-347.8$ & 51.6 & 8.6 \\
\hline $374.3-403.9$ & 95.4 & 5.2 \\
\hline $344.7-314.3$ & 131 & 3.6 \\
\hline $319.3-344.7$ & 157 & $3 \cdot 5$ \\
\hline $294.0-319.3$ & 167 & 3.1 \\
\hline $272.8-294.0$ & 152 & 4.1 \\
\hline $251.7-272.8$ & 133 & 4.4 \\
\hline $234.7-251.7$ & 120 & 5.8 \\
\hline $217.8-234.7$ & 97.4 & 6.7 \\
\hline
\end{tabular}


Table XVI (Cont'd.)

\begin{tabular}{|c|c|c|}
\hline $\begin{array}{c}\text { Energy Interval } \\
(\mathrm{keV})\end{array}$ & $\begin{array}{c}\text { Flux } \\
\text { (neuts } / \mathrm{cm}^{2} / \mathrm{MeV} / \mathrm{min} / \text { watt) }\end{array}$ & $\begin{array}{l}\text { Standard } \\
\text { Error }(\%)\end{array}$ \\
\hline \multicolumn{3}{|c|}{ I Atmosphere Counter } \\
\hline $245.6-300$ & 165 & 5.7 \\
\hline $255.7-275.6$ & 147 & 7.8 \\
\hline $235.8-255.7$ & 119 & 9.0 \\
\hline $218.1-235.8$ & 107 & 10.7 \\
\hline $202.6-218.1$ & 83.2 & 1.4 .9 \\
\hline $18 \% .1-202.6$ & 130 & 8.8 \\
\hline $173.8-187.1$ & 115 & $7 \cdot 3$ \\
\hline $160.5-173.8$ & 164 & $7 \cdot 3$ \\
\hline $147 \cdot 2-160 \cdot 5$ & 159 & 6.9 \\
\hline $136.2-147.2$ & 273 & 4.6 \\
\hline $127 \cdot 3-136.2$ & 268 & 5.7 \\
\hline $116.2-127 \cdot 3$ & 189 & 6.0 \\
\hline $107.4-116.2$ & 119 & 11.0 \\
\hline $100.7=107.4$ & 81.3 & 19.7 \\
\hline $91.9-100 \cdot 7$ & 80.9 & 13.7 \\
\hline $85.2-91.9$ & 113 & 11.8 \\
\hline $78.6-85.2$ & 149 & 8.4 \\
\hline
\end{tabular}


Table XVII. Benfamin Counter Spectrum on the Centerline 10 in. Behind 12 in. of Stainless Steel

\begin{tabular}{|c|c|c|}
\hline $\begin{array}{c}\text { Energy Interval } \\
(\mathrm{keV})\end{array}$ & $\begin{array}{c}\text { Flux } \\
\text { (neutrs } / \mathrm{cm}^{2} / \mathrm{MeV} / \mathrm{min} / \text { wat }\end{array}$ & $\begin{array}{l}\text { Standard } \\
\text { Error }(\%) \\
\end{array}$ \\
\hline \multicolumn{3}{|c|}{10 Atmosphere Counter } \\
\hline $1379 \cdot 3-1500$ & 14.0 & 7.4 \\
\hline $1277 \cdot 1-1379 \cdot 3$ & 19.6 & 6.4 \\
\hline $1184.2-1277.1$ & 28.2 & 5.0 \\
\hline $1091.3-1184.2$ & 32.4 & 4.3 \\
\hline $1017.0=1091.3$ & 36.8 & 5.0 \\
\hline $933.4-1017.0$ & 41.5 & 3.7 \\
\hline $868.4-933.4$ & 40.6 & 5.0 \\
\hline $803.4-868.4$ & 42.8 & 4.5 \\
\hline $738.4-803.4$ & 61.2 & 3.0 \\
\hline $682.7-738.4$ & 88.1 & 2.5 \\
\hline $636.2-682.7$ & 102 & 2.6 \\
\hline $589.8-636.2$ & 112 & 2.3 \\
\hline $543.3-589.8$ & 101 & 2.5 \\
\hline $506.2-543.3$ & 88.9 & 3.4 \\
\hline $459.8-506.2$ & 90.4 & 2.5 \\
\hline $431.9-459.8$ & 102 & 3.5 \\
\hline $394-431.9$ & 124 & 2.1 \\
\hline $366.9-394.7$ & 145 & 2.2 \\
\hline $339.0-366.9$ & 166 & 1.9 \\
\hline \multicolumn{3}{|c|}{3 Atmosphere Counter } \\
\hline $552 \cdot 3-600$ & 109 & 6.0 \\
\hline $513.4-552.3$ & 87.0 & 9.3 \\
\hline $474.4-513.4$ & $84 \cdot 7$ & 9.0 \\
\hline $439.7-474.4$ & 72.4 & 11.2 \\
\hline $405.1-439.7$ & 83.6 & 9.0 \\
\hline $374.7=405.1$ & 140 & 5.9 \\
\hline $344.4-374.7$ & 166 & 4.7 \\
\hline $318.4-344.4$ & 233 & 3.8 \\
\hline $296.8-318.4$ & 243 & $4 \cdot 3$ \\
\hline
\end{tabular}


Table XVII (Cont'd)

\begin{tabular}{|c|c|c|}
\hline $\begin{array}{c}\text { Energy Interval } \\
(\mathrm{keV})\end{array}$ & $\begin{array}{c}\text { Flux } \\
\text { (neuts } / \mathrm{cm}^{2} / \mathrm{MeV} / \mathrm{min} / \text { watt) }\end{array}$ & $\begin{array}{l}\text { Standard } \\
\text { Error }(\%)\end{array}$ \\
\hline $275.1-296.8$ & 254 & 4.0 \\
\hline $253.4-275.1$ & 193 & 5.0 \\
\hline $236.1-253.4$ & 163 & 7.0 \\
\hline $218.8-236.1$ & 137 & 7.7 \\
\hline $201.4-218.8$ & 128 & 7.6 \\
\hline $184.1-201.4$ & 153 & 5.9 \\
\hline $1^{\prime} / 1.1-184.1$ & 1.48 & 7.4 \\
\hline $158.1-171.1$ & 131 & 7.7 \\
\hline $145.1-158.1$ & 223 & 4.2 \\
\hline \multicolumn{3}{|c|}{ I Atmosphere Counter } \\
\hline $230.0-250$ & 173 & 7.4 \\
\hline $212.2-230.0$ & 146 & 9.4 \\
\hline $196.7-212.2$ & 120 & $12 \cdot 3$ \\
\hline $181.1-196.7$ & 190 & 7.2 \\
\hline $167.8-181.1$ & 202 & 7.4 \\
\hline $156.7-167.8$ & 168 & 10.2 \\
\hline $143.3-156.7$ & $25 \bigwedge_{1}$ & 5.1 \\
\hline $134.4-143.3$ & 440 & 4.3 \\
\hline $123 \cdot 3-134 \cdot 4$ & 412 & $3 \cdot 5$ \\
\hline $114 \cdot 4-123 \cdot 3$ & 270 & 6.2 \\
\hline $105.6-114.4$ & 158 & 9.8 \\
\hline $96.7-105.6$ & 136 & 10.5 \\
\hline $90.0-96.7$ & 182 & $9 \cdot 5$ \\
\hline $83.3 .90 \cdot 0$ & 239 & 6.7 \\
\hline
\end{tabular}


Table XVIII. Benjamin Counter Spectrum 12 in. Off the Centerline and $10 \mathrm{in.}$ Behind 12. in. of Stainless Steel

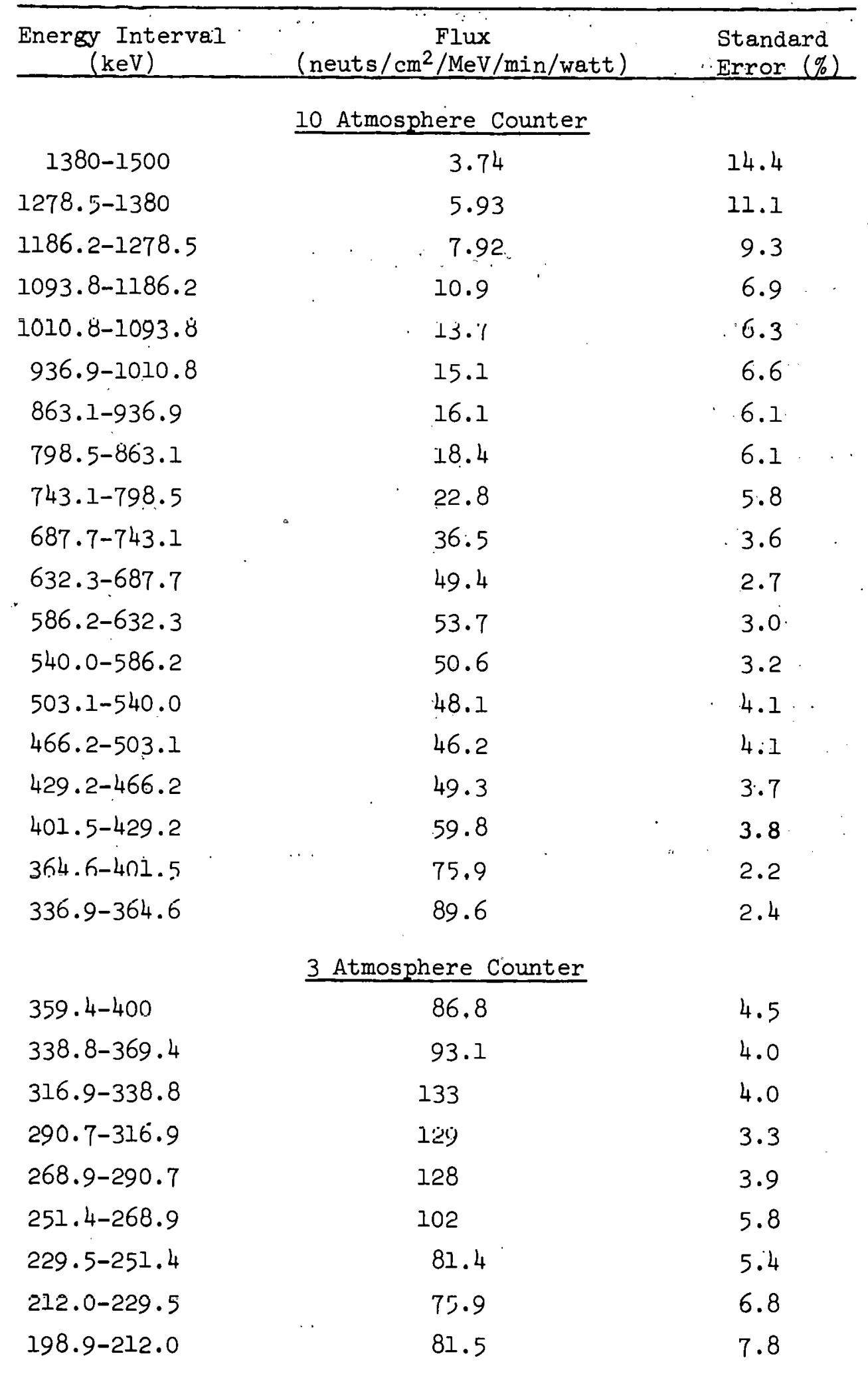


Table XVIII (Cont'd.)

\begin{tabular}{|c|c|c|}
\hline $\begin{array}{l}\text { Energy Interval } \\
(\mathrm{keV})\end{array}$ & $\begin{array}{c}\text { Flux } \\
\text { (neuts } / \mathrm{cm}^{2} / \mathrm{MeV} / \mathrm{min} / \text { watt) }\end{array}$ & $\begin{array}{l}\text { Standard } \\
\text { Error }(\%)\end{array}$ \\
\hline $181.4-198.9$ & 93.7 & 4.8 \\
\hline $168.3-181.4^{\circ}$ & 86.5 & 6.4 \\
\hline $155.2-168.3$ & 96.1 & 5.3 \\
\hline $146.4-155.2$ & 162 & 4.2 \\
\hline & 1 Atmosphere Counter & \\
\hline $230.0-250$ & 112 & 8.1 \\
\hline $212.2-230.0$ & 88.9 & 10.9 \\
\hline $196.1-x^{\prime}+\perp^{\prime}{ }^{\prime} .2$ & 73.5 & $14 \cdot 3$ \\
\hline $181.1-196.7$ & 100 & 9.7 \\
\hline $167.8-181.1$ & 114 & 9.4 \\
\hline $156.7-167.8$ & 98.7 & 12.4 \\
\hline $143.3-156.7$ & 147 & 6.3 \\
\hline $134.4-143.3$ & 244 & 5.6 \\
\hline $123 \cdot 3-134 \cdot 4$ & 240 & 4.3 \\
\hline $114.4-123 \cdot 3$ & 182 & 6.7 \\
\hline $105.6-114.4$ & 107 & 10.6 \\
\hline $96.7-105.6$ & 70.5 & $14 \cdot 7$ \\
\hline $90.0-96.7$ & 200 & 12.6 \\
\hline $83.3-90.0$ & 150 & 7.8 \\
\hline
\end{tabular}


Table XIX. NE-213 Spectrum 15 Deg off the Centerline Behind 4 in. of Iron

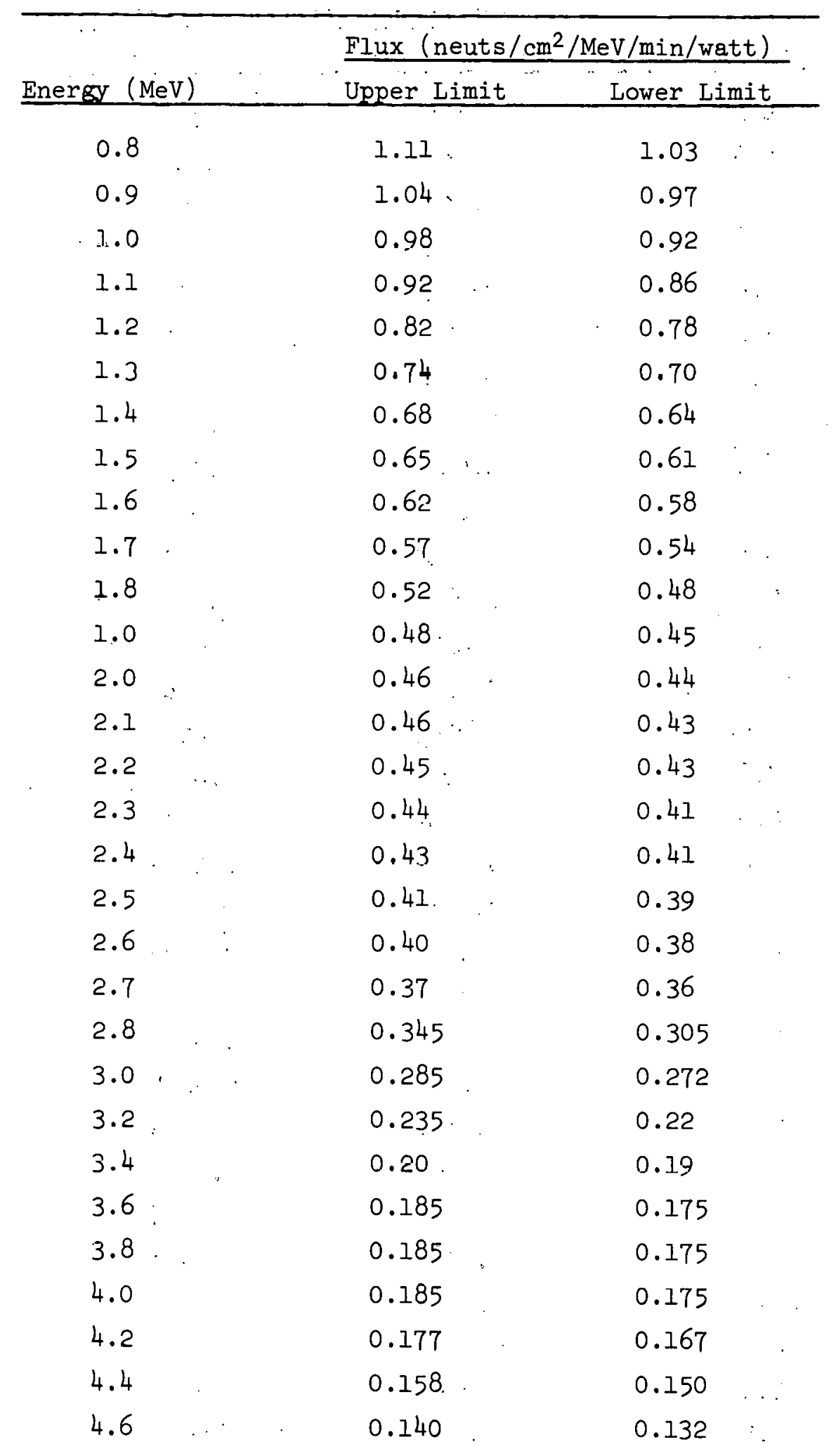


Table XIX (Cont'd.)

\begin{tabular}{cll}
\hline & \multicolumn{2}{c}{ Flux (neuts $/ \mathrm{cm}^{2} / \mathrm{MeV} / \mathrm{min} /$ watt $)$} \\
\hline Energy (MeV) & Upper Limit & Lower Limit \\
\hline 4.8 & 0.128 & 0.120 \\
5.0 & 0.120 & 0.113 \\
5.2 & 0.107 & 0.100 \\
5.4 & 0.092 & 0.085 \\
5.6 & 0.082 & 0.076 \\
5.8 & 0.079 & 0.073 \\
6.0 & 0.076 & 0.070 \\
6.2 & 0.070 & 0.064 \\
6.4 & 0.064 & 0.059 \\
6.6 & 0.060 & 0.0545 \\
6.8 & 0.0535 & 0.049 \\
7.0 & 0.045 & 0.041 \\
7.2 & 0.037 & 0.033 \\
7.4 & 0.032 & 0.028 \\
7.6 & 0.0283 & 0.0248 \\
7.8 & 0.026 & 0.0225 \\
8.0 & 0.0232 & 0.020 \\
8.2 & 0.020 & 0.0173 \\
8.4 & 0.0177 & 0.015 \\
8.6 & 0.016 & 0.0133 \\
8.8 & 0.0144 & 0.0119 \\
9.0 & 0.013 & 0.010 \\
9.2 & 0.0117 & 0.0094 \\
9.4 & 0.0107 & 0.0086 \\
9.6 & 0.0100 & 0.0080 \\
9.8 & 0.0094 & 0.0076 \\
10.0 & 0.0086 & 0.0069 \\
10.2 & 0.0077 & 0.0060 \\
10.4 & 0.0067 & 0.0052 \\
10.6 & 0.0057 & 0.0043 \\
10.8 & 0.0046 & 0.0032 \\
11.0 & 0.0033 & 0.0019 \\
\hline
\end{tabular}


Table XX. NE-213 Spectrum 45 Deg off the:Centerline Behind 4 in. of Iron

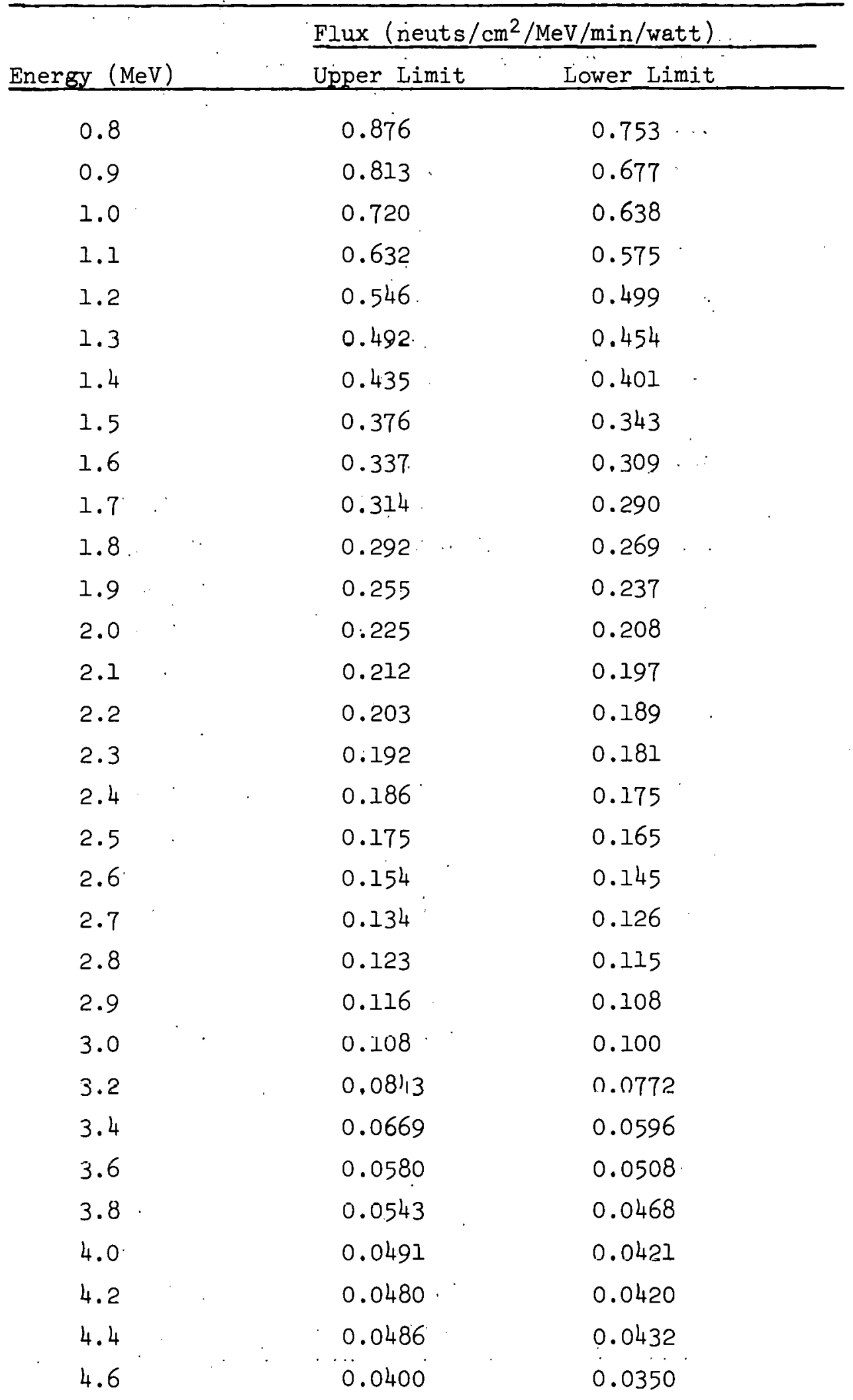


Table XX (Cont'd.)

\begin{tabular}{|c|c|c|}
\hline \multirow{2}{*}{ Energy $(\mathrm{MeV})$} & \multicolumn{2}{|c|}{ Flux (neuts $/ \mathrm{cm}^{2} / \mathrm{MeV} / \mathrm{min} /$ watt) } \\
\hline & Upper Limit & Lower Limit \\
\hline 4.8 & 0.0296 & 0.0252 \\
\hline 5.0 & 0.0252 & 0.0210 \\
\hline 5.2 & 0.0244 & 0.0203 \\
\hline 5.4 & 0.0225 & 0.0189 \\
\hline 5.6 & 0.0190 & 0.0156 \\
\hline 5.8 & 0.0153 & 0.0121 \\
\hline 6.0 & 0.0145 & 0.0114 \\
\hline 6.2 & 0.0110 & 0.0111 \\
\hline 6.4 & 0.0124 & 0.00962 \\
\hline 6.6 & 0.0104 & 0.00780 \\
\hline 6.8 & 0.00931 & 0.00703 \\
\hline 7.0 & 0.00856 & 0.00650 \\
\hline $7 \cdot 2$ & $0.006 \% 4$ & 0.00464 \\
\hline 7.4 & 0.00564 & 0.00376 \\
\hline 7.6 & 0.00582 & 0.00414 \\
\hline 7.8 & 0.00501 & 0.00334 \\
\hline 8.11 & 0.00312 & 0.010 .53 \\
\hline 8.2 & 0.00226 & 0.00098 \\
\hline 8.4 & 0.00233 & 0.00109 \\
\hline 8.6 & 0.00223 & 0.00108 \\
\hline 8.8 & 0.00235 & 0.00130 \\
\hline 9.0 & 0.00271 & 0.00172 \\
\hline $9 \cdot 2$ & 0.00256 & 0.00163 \\
\hline 9.4 & 0.00190 & 0.00098 \\
\hline 9.6 & 0.00133 & 0.00052 \\
\hline 9.8 & 0.00113 & 0.00039 \\
\hline 10.0 & 0.00100 & 0.00023 \\
\hline 10.2 & 0.00080 & 0.00005 \\
\hline 10.4 & 0.00075 & 0.00001 \\
\hline 10.6 & 0.00082 & 0.00006 \\
\hline 10.8 & 0.00079 & 0.00006 \\
\hline
\end{tabular}


Table XXI. NE-213 Spectrum on the Centerline Behind 6 in. of Iron

\begin{tabular}{|c|c|c|c|}
\hline \multirow[b]{2}{*}{ Energy $(\mathrm{MeV})$} & 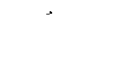 & \multicolumn{2}{|c|}{ Flux (neuts $/ \mathrm{cm}^{2} / \mathrm{MeV} / \mathrm{min} /$ watt) } \\
\hline & c & Upper Limit & Lower Limit \\
\hline 0.8 & & 24.7 & 22.9 \\
\hline 0.9 & & 23.6 & 21.8 \\
\hline 1.0 & & 22.6 & 21.3 \\
\hline 1.1 & & 22.1 & 21.0 \\
\hline 1.2 & & 21.0 & 20.2 \\
\hline 1.3 & & 19.0 & 18.3 \\
\hline 1.4 & & 17.1 & 16.3 \\
\hline 1.5 & & 15.5 & 14.8 \\
\hline 1.6 & & 14.3 & 13.8 \\
\hline 1.7 & & 13.4 & 12.9 \\
\hline 1.8 & & 12.2 & 11.7 \\
\hline 1.9 & . & 11.0 & 10.4 \\
\hline 2.0 & & 9.8 & 9.4 \\
\hline 2.1 & & 9.0 & 8.6 \\
\hline 2.2 & & 8.2 & 7.9 \\
\hline 2.3 & & $7 \cdot 5$ & $7 \cdot 3$ \\
\hline 2.4 & & 6.85 & 6.6 \\
\hline $2 \cdot 5$ & & 6.2 & 6.0 \\
\hline 2.6 & & 5.65 & $5 \cdot 55$ \\
\hline 2.7 & & 5.15 & 5.0 \\
\hline 2.8 & & 4.65 & $4 \cdot 55$ \\
\hline 2.9 & & 4.2 & 4.1 \\
\hline 3.0 & & 3.8 & 3.7 \\
\hline 3.2 & & 2.97 & 2.86 \\
\hline 3.4 & & 2.32 & 2.23 \\
\hline 3.6 & & 1.90 & 1.82 \\
\hline 3.8 & & 1.60 & 1.55 \\
\hline 4.0 & & 1.45 & 1.38 \\
\hline 4.2 & & 1.30 & 1.25 \\
\hline 4.4 & & 1.18 & 1.14 \\
\hline 4.6 & & 1.07 & 1.03 \\
\hline
\end{tabular}


Table XXI (Cont'd.)

Flux (neuts $/ \mathrm{cm}^{2} / \mathrm{MeV} / \mathrm{min} /$ watt)

Energy (MeV) Upper Limit Lower Limit

\begin{tabular}{|c|c|c|}
\hline 4.8 & 0.945 & 0.90 \\
\hline 5.0 & 0,82 & 0.74 \\
\hline 5.2 & 0.68 & 0.64 \\
\hline 5.4 & 0.605 & 0.57 \\
\hline 5.6 & 0.56 & 0.52 \\
\hline 5.8 & 0.52 & 0.185 \\
\hline 6.0 & 0.49 & 0.455 \\
\hline 6.2 & 0.44 & 0.415 \\
\hline 6.4 & 0.39 & 0.36 \\
\hline 6.6 & 0.345 & 0.32 \\
\hline 6.8 & 0.325 & 0.297 \\
\hline 7.0 & 0.300 & 0.275 \\
\hline 7.2 & 0.268 & 0.243 \\
\hline 7.4 & 0.222 & 0.200 \\
\hline 7.6 & 0.190 & 0.170 \\
\hline 7.8 & 0.175 & 0.155 \\
\hline 8.0 & 0.165 & 0.147 \\
\hline 8.2 & 0.154 & 0.137 \\
\hline 8.4 & 0.143 & 0.126 \\
\hline 8.6 & 0.134 & 0.117 \\
\hline 8.8 & 0.124 & 0.108 \\
\hline 9.0 & 0.113 & 0.098 \\
\hline 9.2 & 0.104 & 0.089 \\
\hline 9.4 & 0.095 & 0.080 \\
\hline 9.6 & 0.084 & 0.010 \\
\hline 9.8 & 0.071 & 0.058 \\
\hline 10.0 & 0.060 & 0.047 \\
\hline 10.2 & 0.050 & 0.038 \\
\hline 10.4 & 0.042 & 0.031 \\
\hline 10.6 & 0.039 & 0.028 \\
\hline 10.8 & 0.041 & 0.029 \\
\hline 11.0 & 0.044 & 0.033 \\
\hline 11.2 & 0.046 & 0.035 \\
\hline
\end{tabular}


Table XXII. NE-213 Spectrum on the Centerline Behind 12 in. of Iron

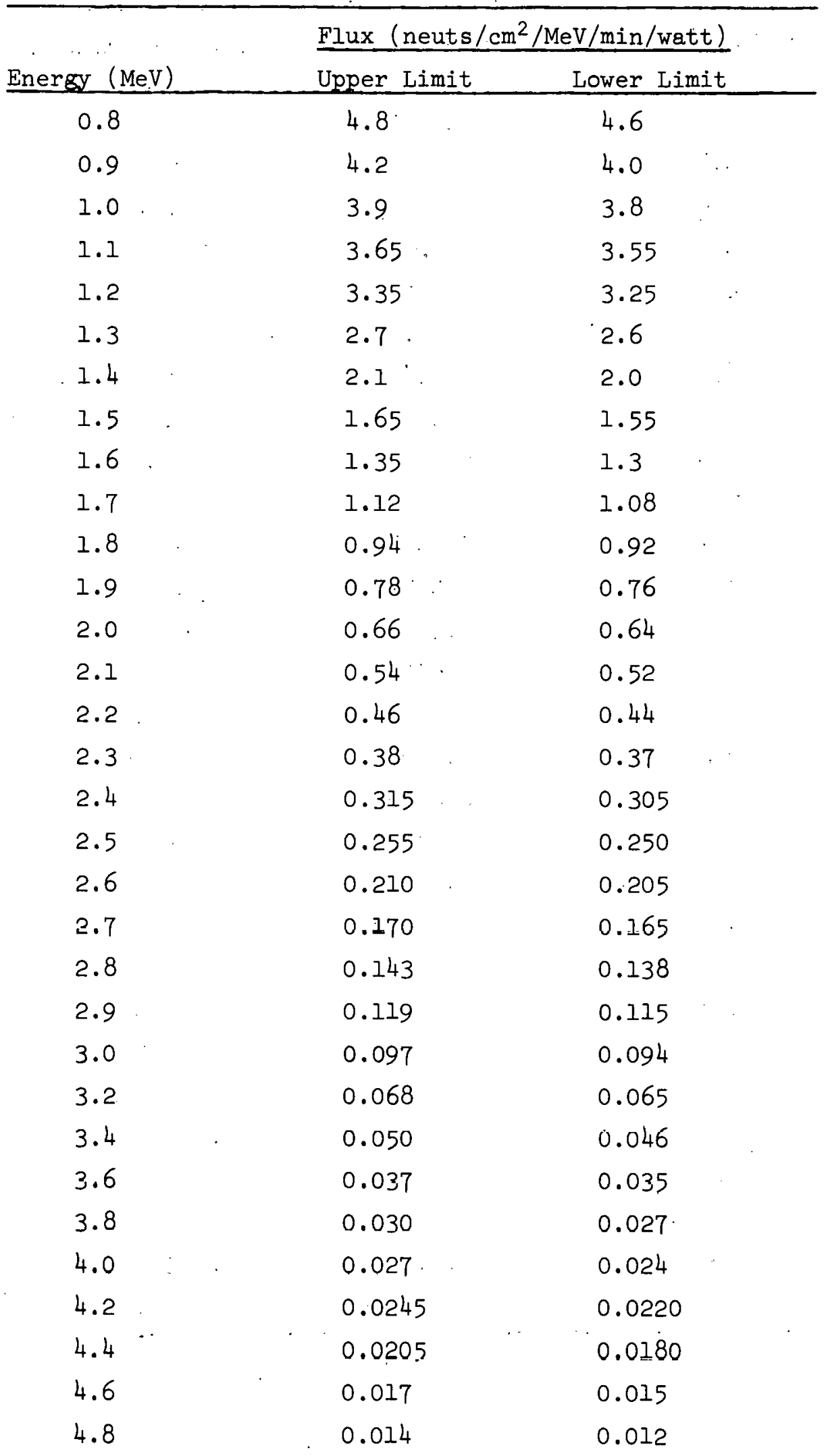


Table XXII (Cont'd.)

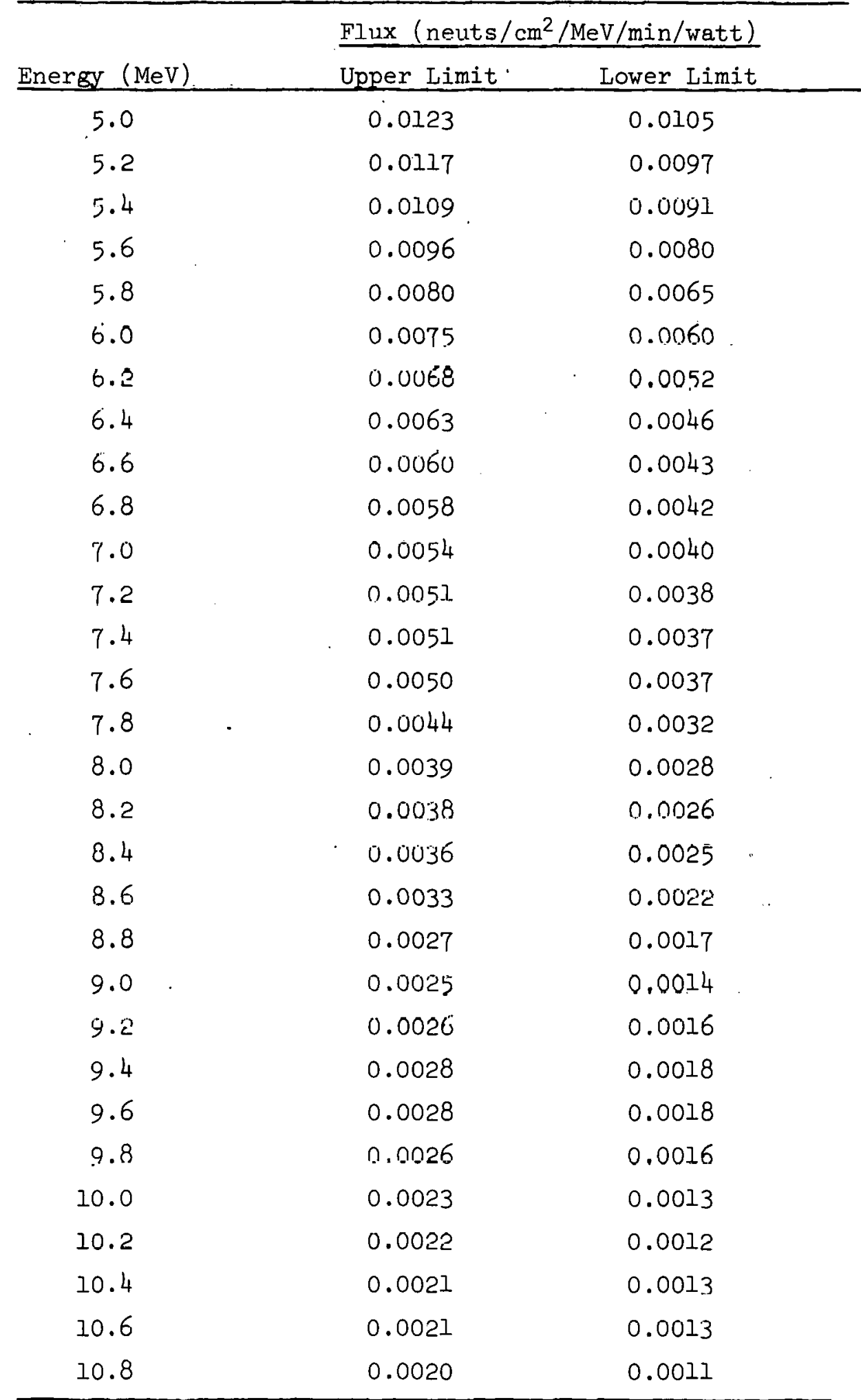


Table XXIII. NE-213 Spectrum 15 Deg off the Centerline .. Behind 12 in. of Iron

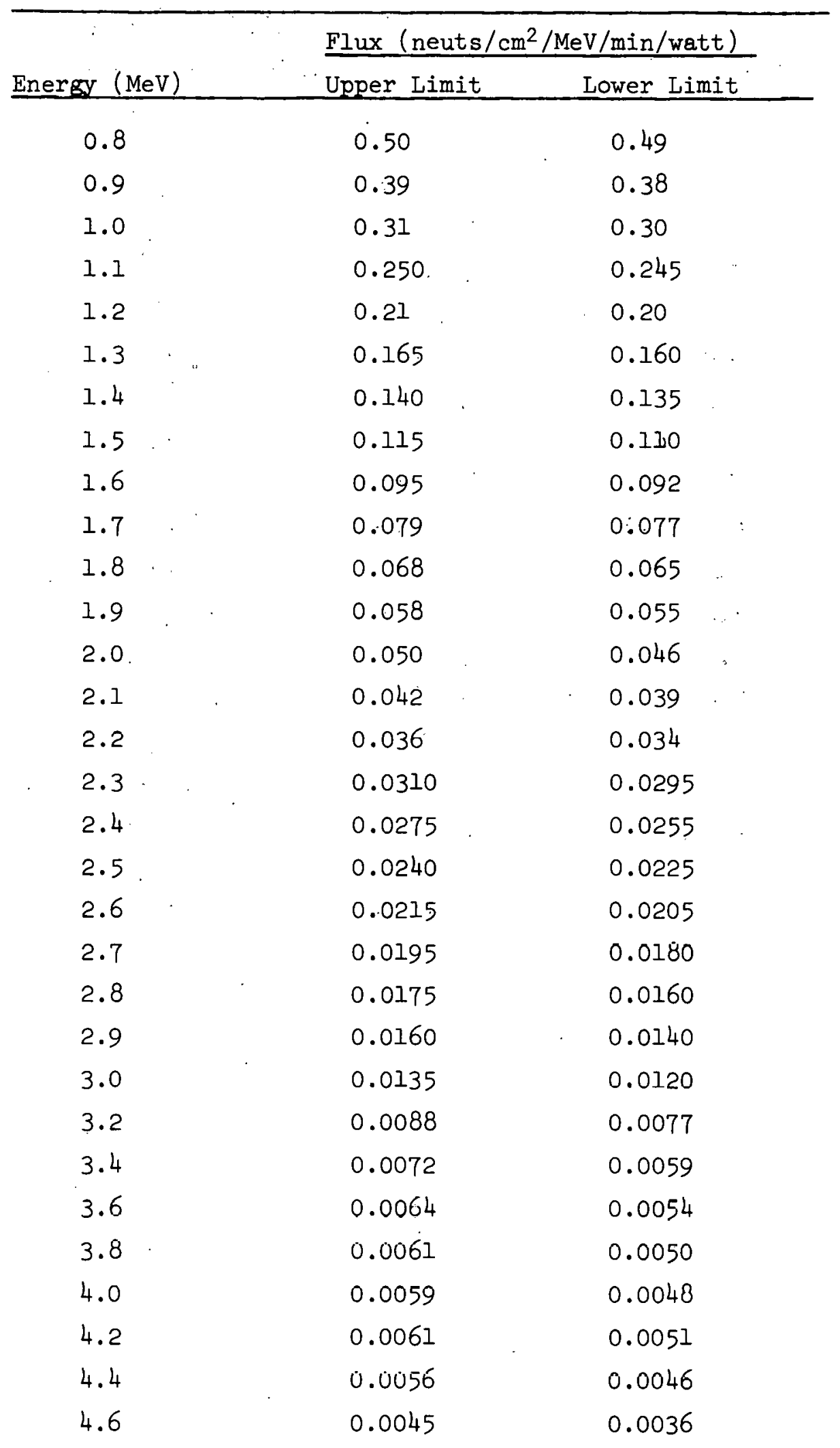


Table XXIII (Cont'd.)

\begin{tabular}{|c|c|c|}
\hline \multirow[b]{2}{*}{ Energy (MeV) } & \multicolumn{2}{|c|}{ Flux (neuts $/ \mathrm{cm}^{2} / \mathrm{MeV} / \mathrm{min} /$ watt) } \\
\hline & Upper Limit & Lower Limit \\
\hline 4.8 & 0.0037 & 0.0028 \\
\hline 5.0 & 0.0032 & 0.0025 \\
\hline 5.2 & 0.00270 & 0.00215 \\
\hline 5.4 & 0.00265 & 0.00195 \\
\hline 5.6 & 0.00265 & ח. .0อ?חก \\
\hline 5.8 & 0.0030 & 0.0123 \\
\hline 6.0 & 0.0031 & 0.0024 \\
\hline 6.2 & 0.00260 & 0.00195 \\
\hline 6.4 & 0.0020 & 0.0014 \\
\hline 6.6 & 0.0017 & 0.0011 \\
\hline 6.8 & 0.00160 & 0.00105 \\
\hline 7.0 & 0.00155 & 0.00102 \\
\hline 7.2 & 0.00155 & 0.00100 \\
\hline 7.4 & 0.00148 & 0.00098 \\
\hline 7.6 & 0.00135 & 0.00089 \\
\hline 7.8 & 0.00117 & 0.00076 \\
\hline 8.0 & 0.00108 & 0.00066 \\
\hline 8.2 & 0.00110 & 0.00068 \\
\hline 8.4 & 0.00117 & 0.00076 \\
\hline 8.6 & 0.00120 & 0.00081 \\
\hline 8.8 & 0.00110 & 0.00070 \\
\hline
\end{tabular}


Table XXIV. NE-213 Spectrum 45 Deg off the Centerline Behind 12 in. of Iron

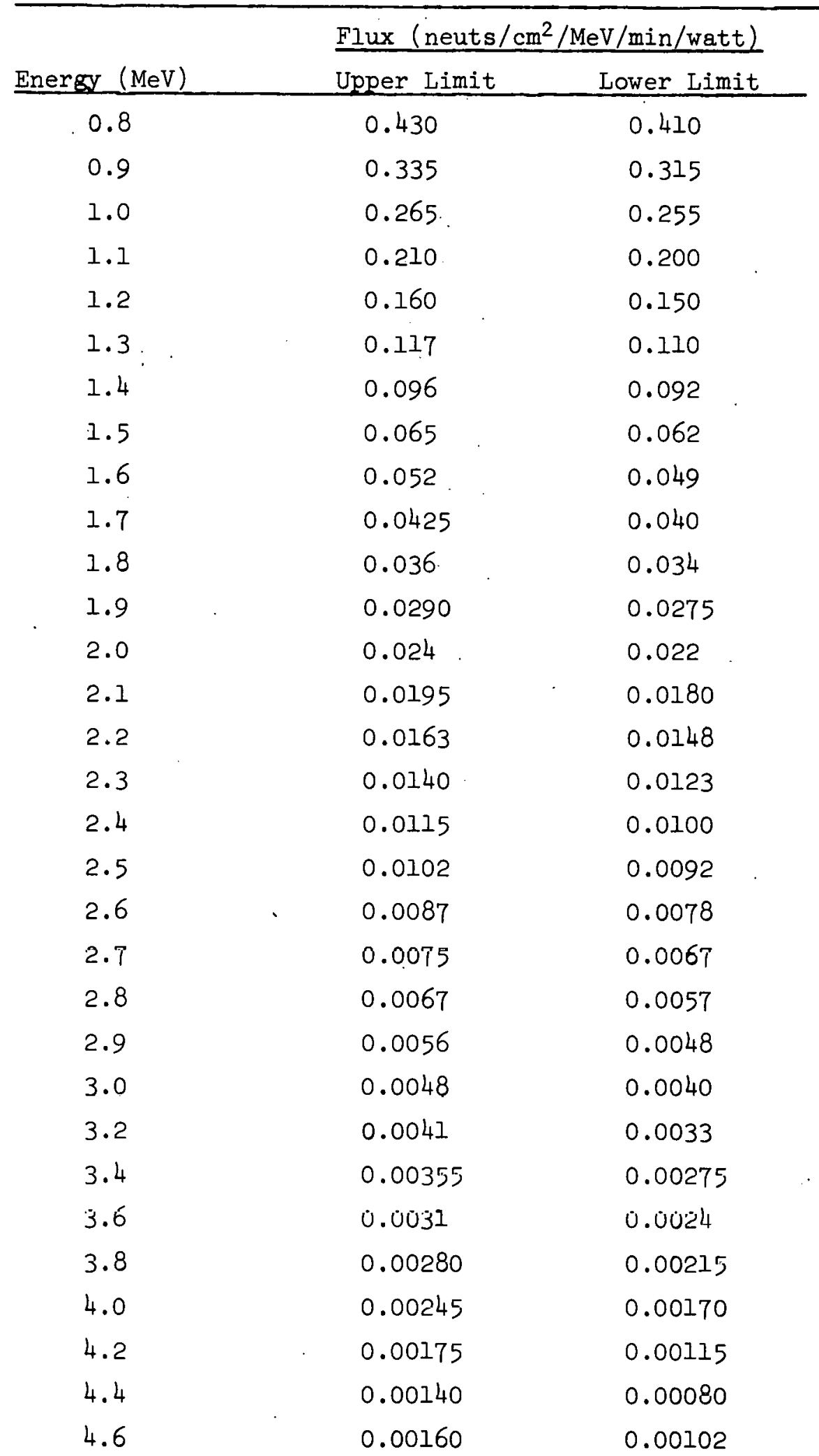


Table XXIV (Cont'd.)

\begin{tabular}{ccc} 
& \multicolumn{2}{c}{ Flux (neuts $/ \mathrm{cm}^{2} / \mathrm{MeV} / \mathrm{min} /$ watt) } \\
\cline { 2 - 3 } Energy (MeV) & Upper Limit & Lower Limit \\
\hline 4.8 & 0.00170 & 0.00110 \\
5.0 & 0.00146 & 0.00090 \\
5.2 & 0.00124 & 0.00066 \\
5.4 & 0.00090 & 0.00051 \\
5.6 & 0.00079 & 0.00034 \\
5.8 & 0.00071 & 0.00026 \\
6.0 & 0.00076 & 0.00033 \\
6.2 & $0.0008 \%$ & 0.00039 \\
6.4 & 0.00068 & 0.00029 \\
6.6 & 0.00052 & 0.00012 \\
6.8 & 0.00044 & 0.00006 \\
7.0 & 0.00048 & 0.00012 \\
7.2 & 0.00050 & $0.0001 \%$ \\
7.4 & 0.00040 & 0.00011 \\
\hline
\end{tabular}


Table XXV. Approximate Enhancement of the Transmitted Slab Scattered Flux Above Thermal Energy Arising from Multiple Reflection in the Collimator

$\phi_{T}$ Including Collimator $/ \phi_{\mathrm{T}}$ Not Including Collimator

$1-1 / 2$ in. of Iron

12 in. of Iron

24 in. of Iron

36 in. of Iron

12 in. of Stainless Steel
1.11

1.21

1.21

1.10

1.17 
Table XXVI. Comparison of 220-Group and 100-Group (GAM-II) ANISN Results of Leakage from 1-Meter-Fudiuy Iron Sphere Arising from Point P1saion Source Locsted at the Center

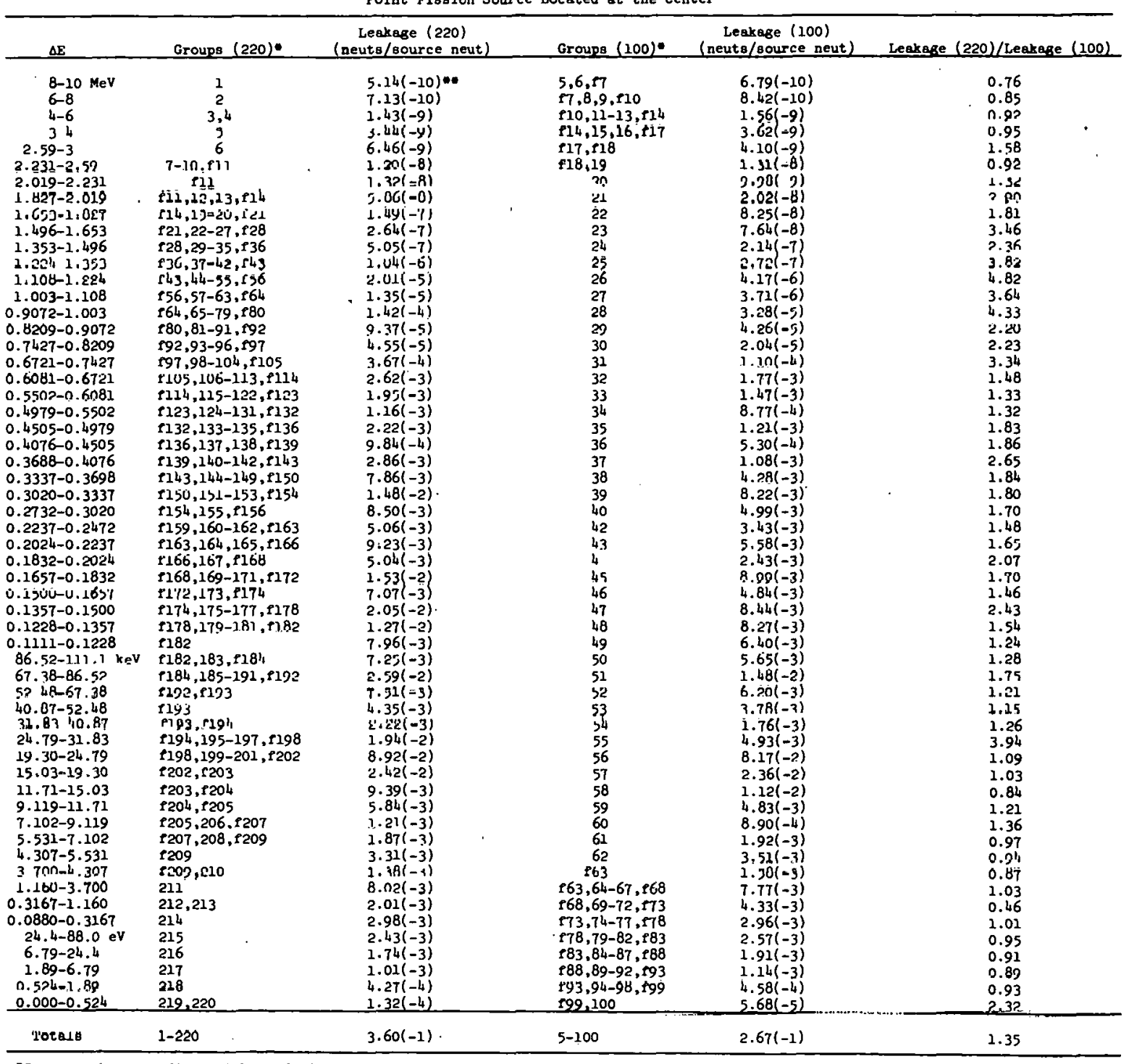

-Group numbers prefixed with an fle in more than one energy fnterval $\Delta E$.

mead as $5.14 \times 10^{-10}$, etr. 
ORNL-TM-4222

(ENDF 188)

INTERNAL DISTRIBUTION

$\begin{aligned} & \text { 1-20. } \text { L. S. Abbott } \\ & 21 . \text { C. E. Clifford } \\ & 22 . \text { W. O. Harms } \\ & 23 . \text { R. E. Maerker } \\ & 24 . \text { F. C. Maiens chein } \\ & \text { 25. F. R. Mynatt } \\ & \text { 26. E. M. Oblow } \\ & \text { 27. } \\ & \text { D. B. Trauger } \\ & \text { 28. D. K. Trubey } \\ & \text { 30. H. E. Whites Ldes } \\ & \text { 31. P. F. Fox (consultant) } \\ & \text { 32. W. W. Havens (consultant) } \\ & \text { 33. A. F. Henry (consultant) }\end{aligned}$

34-35. Central Research Library

36-37. ORNL Y-12 Technical Library Document Reference Section

38-43. Laboratory Records

44. Laboratory Records ORNL RC

45. ORNL Patent Office

46-96. Radiation Shielding Information Center

EXTERNAL DISTRIBUTION

97-146. Technical Information Center for ENDF distribution 147-148. Technical Information Center

149. Research and Technical Support Division 